\title{
EQUISINGULAR DEFORMATIONS OF PLANE ALGEBROID CURVES(1)
}

\author{
BY
}

\author{
JONATHAN M. WAHL
}

\begin{abstract}
We construct a formal versal equisingular deformation of a plane algebroid curve (in characteristic zero), and show it is smoothly embedded in the whole deformation space of the singularity. Closer analysis relates equisingular deformations of the curve to locally trivial deformations of a certain (nonreduced) projective curve. Finally, we prove that algebraic $\pi_{1}$ of the complement of a plane algebroid curve remains constant during formal equisingular deformation. .
\end{abstract}

Introduction. In a series of papers ([10], [11], [12]), Zariski has studied the concept of equisingularity of plane algebroid curves. Two curves are equisingular if one can simultaneously resolve their singularities; this equivalence relation is weaker than analytic equivalence, but stronger than equimultiplicity. Using topological techniques, Zariski proves that two equisingular curves over $\mathbf{C}$ have locally the same topological embedding in $\mathbf{C}^{2}$; in particular, the characteristic pairs of their branches are the same, whence they yield knots of the same knot type in $\mathbf{R}^{3}$ (cf. [4]).

Utilizing techniques developed by M. Schlessinger [6], we study infinitesimal equisingular families of curves. Our deformation theory takes place over the category $\underline{C}$ of artin local C-algebras. Recall that if $f \in \mathbf{C}[[X, Y]]$ is reduced, and if $g_{1}, \cdots, g_{m} \in \mathrm{C}[[X, Y]]$ induce a basis of the artin ring $\mathrm{C}[[X, Y]] /\left(f, f_{X}, f_{Y}\right)$, then the formal family $f+t_{1} g_{1}+\cdots+t_{m} g_{m} \in \mathbf{C}\left[\left[X, Y, t_{1}, \cdots, t_{m}\right]\right]$ induces a formal versal (or semiuniversal) deformation of the singularity defined by $(f)$. Thus, in a weak sense, the family represents the functor on $\underline{C}$ of infinitesimal deformations of the singularity.

To define equisingular deformation, we emulate Zariski's original definition. Recall that every plane algebroid singularity can be reduced to a number of ordinary double points by a finite number of quadratic transforms. We say a deformation

Received by the editors December 19, 1972.

AMS (MOS) subject classifications (1970). Primary 14D15, 14H20; Secondary 14B10, $32 \mathrm{C} 40$.

Key words and phrases. Plane algebroid curve, equisingularity, deformation space, infinitesimal deformation, algebraic fundamental group.

(1) Research partially supported by NSF Research Grant GP-20532. 
$A[[X, Y]] /(\bar{f})(A \in \underline{C})$ is equisingular if it is normally flat (i.e., equimultiple) along an $A$-section and, after blowing up the section, the transform is equisingular along $A$-sections lying above the first; finally, equisingular means equimultiple for an ordinary double point. Theorems 3.2 and 4.2 below imply

Theorem. There exists a smooth closed subscheme of Spec C $\left.\left[t_{1}, \cdots, t_{m}\right]\right]$ on which the induced family of curves yields a formal versal equisingular deformation. Infinitesimal equisingular sections are unique.

There are some equisingular deformations that are simpler than others, namely those for which all significant equisingular sections can be simultaneously trivialized; call the functor of such deformations $E S^{\prime}$, a subfunctor of the regular equisingular functor $E S$. From most points of view, $E S^{\prime}$ is much easier to work with. For instance, the tangent space of $E S^{\prime}$ is easily described algebraically (Proposition 6.3), and in $\$ 8$ we prove

Theorem. $E S^{\prime}=E S$ if and only if, for appropriate choice of $g_{1}, \cdots, g_{m}$, the subscheme of the previous theorem may be given by $t_{1}=\cdots=t_{r}=0$; that is, there is a versal equisingular deformation defined by $f+\sum_{i=r+1}^{m} t_{i} g_{i}$.

Using this result, we show a formal versal equisingular deformation of $Y^{p}+X^{q}$ is given by $Y^{p}+X^{q}+\Sigma t_{i j} X^{i} Y^{j}$, where the sum is over pairs $(i, j)$ with $i \leq q-2, j \leq p-2$, and $(i / q)+(i / p) \geq 1$. On the other hand, $E S^{\prime}$ is in general smaller than ES (Example 6.8); in fact, $E S^{\prime}$ is the kernel of a smooth morphism $E S \rightarrow L$, where $L$ may be viewed as the functor of classes of locally trivial deformations of a certain nonreduced projective curve (Theorem 5.7).

Finally, using a theorem on deformations of branched coverings from [8], we prove, in $\$$,

Theorem. Let $F\left(X, Y, t_{1}, \cdots, t_{n}\right) \in \mathbf{C}\left[\left[X, Y, t_{1}, \cdots, t_{n}\right]\right]$ define a formal equisingular deformation of $F(X, Y, 0, \ldots, 0)=f(X, Y)$. Then the natural map

$$
\pi_{1}(\operatorname{Spec} \mathrm{C}[[X, Y]]-V(f)) \rightarrow \pi_{1}\left(\operatorname{Spec} \mathrm{C}\left[\left[X, Y, t_{1}, \cdots, t_{n}\right]\right]-V(F)\right)
$$

is an isomorphism.

Here, $\pi_{1}$ is the algebraic fundamental group. Though this result is weaker than Zariski's, the proof is purely algebraic, and is established (as are all our results) for any algebraically closed field $k$ of characteristic zero. The proof utilizes the Grothendieck existence theorem and a well-known result on the algebraicity of the ring $k[[X, Y]] /(f)$.

A portion of this paper appeared in the author's 1971 Harvard thesis, Deformations of branched covers and equisingularity. We are grateful to Bruce Bennett and Michael Schlessinger for several useful conversations, and are indebted to David Mumford for his advice and support. 


\section{Preliminaries.}

(0.1) We shall rely on the techniques of functors of artin rings. Let $\underline{C}$ be the category of artin local $k$-algebras; denote by $k[\epsilon]$ the object $k[\epsilon] / \epsilon^{2}$. Every surjection in $\underline{C}$ may be factored by small extensions, i.e., ones whose kernel is onedimensional over $k$.

(0.2) Let $F$ be a covariant functor from $\underline{C}$ to sets with $F(k)=$ one point. If $A^{\prime} \rightarrow A$ and $A^{\prime \prime} \rightarrow A$ are morphisms in $\underline{C}$, there is a natural map

$$
(\alpha): F\left(A^{\prime} \times_{A} A^{\prime \prime}\right) \rightarrow F\left(A^{\prime}\right) \times_{F(A)} F\left(A^{\prime \prime}\right) \text {. }
$$

Recall the conditions of Schlessinger [6]:

$\left(\mathrm{H}_{1}\right)(a)$ is a surjection when $A^{\prime} \rightarrow A$ is small.

$\left(\mathrm{H}_{2}\right)(a)$ is a bijection when $A^{\prime}=k[\epsilon], A=k$.

$\left(\mathrm{H}_{3}\right) F(k[\epsilon])$ (the tangent space) is a finite-dimensional $k$-vector space.

$\left(\mathrm{H}_{4}\right)(a)$ is a bijection when $A^{\prime} \rightarrow A$ is small.

We say that $F$ satisfying $\left(\mathrm{H}_{1}\right)$ and $\left(\mathrm{H}_{2}\right)$ has a good deformation theory; if it satisfies $\left(\mathrm{H}_{4}\right)$ as well, we say it has a very good deformation theory. If $F$ has a good deformation theory and $A^{\prime} \rightarrow A$ is small, then $F(k[\epsilon])$ is a $k$-vector space and it acts transitively on the fibres of $F\left(A^{\prime}\right) \rightarrow F(A)$; if $F$ has a very good theory, the action is free as well. $F$ is smooth if $F\left(A^{\prime}\right) \rightarrow F(A)$ is always surjective.

(0.3) Schlessinger's criterion [6] says that a functor with a good (respectively very good) deformation theory satisfying $\left(\mathrm{H}_{3}\right)$ is versal (resp. pro-representable); that is, there is a complete local $k$-algebra $R$, of residue field $k$, and a morphism $b_{R} \rightarrow F$ which is smooth and a bijection on the tangent spaces (resp. which is a bijection). We refer the reader to [8] for a discussion and examples.

\section{Equimultiple liftings.}

(1.1) Let $f \in k[[X, Y]]=R$ be a reduced formal power series of degree $r \geq 1$, i.e., $f \in(X, Y)^{r}-(X, Y)^{r+1}$. Denote by $f_{r}$ the leading form of $f$; the linear factors of $f_{r}$ define the tangent directions of $V(f) \subset$ Spec $R$. By Hensel's lemma, one knows that $f$ irreducible implies $f_{r}=(a X+b Y)^{r}$, for some $a, b \in k$; hence $f$ irreducible implies $f$ unitangential (has one tangent direction). Thus, any $f$ may be written as a product of tangential components.

(1.2) We consider several important functors on $\underline{C}$ :

$$
\begin{aligned}
H(A) & =\left\{\text { ideals }(\bar{f}) \subset R \otimes_{k} A=R_{A} \mid \bar{f} \text { induces } f \text { in } R\right\} \\
& =\text { set of (flat) liftings of }(f) \text { to } R_{A} \cdot \\
G(A) & =\text { groups of } A \text {-automorphisms of } R_{A} \text { inducing the identity on } R .
\end{aligned}
$$

$H$ and $G$ have very good deformation theories and are smooth; the quotient $D$ of $H$ by the natural action of $G$ is versal and smooth, and is the functor of deformation classes of the algebra $R /(f)[8]$.

(1.3) Denote by $s: R /(f) \rightarrow k$ the residue field map. Recall that a surjection 
of rings $S \rightarrow T=S / l$ is normally flat if $l^{p} / I^{p+1}$ is a locally free $T$-module for all $p \geq 1$. We define a functor $E$ on $\underline{C}$ by

$$
\begin{aligned}
E(A)=\{((\bar{f}), \bar{s}) \mid(\bar{f}) & \in H(A) \text { and } \\
& \left.\bar{s}: R_{A} /(\bar{f}) \rightarrow A \text { is a normally flat } A \text {-section inducing } s\right\} .
\end{aligned}
$$

Given $(\bar{f})$, the section $\bar{s}$ is uniquely determined by an ideal $\bar{I} \subset R_{A}$, with $(\bar{f}) \subset$ $\bar{I}$; $\bar{I}$ may be written $\left(X-m_{1}, Y-m_{2}\right)$, for some $m_{i} \in m_{A}$. The section $\bar{s}$ is called trivial if $\bar{I}=(X, Y)$. We shall eventually show $E$ has a very good deformation theory and is smooth.

Lemma 1.4. Let $A^{\prime} \rightarrow A$ be small, with kernel $(\epsilon)$, and let $((\bar{f}), \bar{s}) \in E(A)$, where $\bar{s}$ is defined by the ideal $\bar{I} \subset R_{A}$. Suppose $\left(f^{\prime}\right) \in H\left(A^{\prime}\right)$ lifts $(\bar{f})$ and $I^{\prime} \epsilon$ $R_{A^{\prime}}$ lifts $\bar{I}$, and defines a section $s^{\prime}: R_{A^{\prime}} /\left(f^{\prime}\right) \rightarrow A^{\prime}\left(\right.$ i.e., $\left.\left(f^{\prime}\right) \subset l^{\prime}\right)$. Then $\left(\left(f^{\prime}\right), s^{\prime}\right) \in E\left(A^{\prime}\right)$ if and only if $g \in I^{p}$ and $\epsilon g \in\left(I^{\prime p+1}, f^{\prime}\right)$ imply $g \in\left(I^{p+1}\right.$, $\left.f^{\prime}, m_{A^{\prime}} I^{\prime p}\right)$, for all integers $p \geq 1$.

Proof. This is essentially Grothendieck's infinitesimal criterion of flatness [1, IV. 5].

(1.5) There is a functorial group action $G \times E \rightarrow E$ defined by

$$
\sigma((\bar{f}), \bar{s})=\left(\sigma(\bar{f}), \bar{s} \cdot \sigma^{-1}\right),
$$

with obvious notation; one easily checks that $\sigma(\bar{f})$ is normally flat along $\bar{s} \cdot \sigma^{-1}$, and that the action is indeed a functorial group action. Note that, for every $\eta \epsilon$ $E(A)$, there is a $\sigma \in G(A)$ such that $\sigma(\eta)$ has a trivial section.

The following result shows that $E$ is the functor of equimultiple liftings and sections; we shall subsequently drop the term normally flat.

Proposition 1.6. For $A \in \underline{C}$, let $m_{1}, \cdots, m_{u}$ be a $k$-basis for $m_{A}$, and let $(\bar{f}) \in H(A)$, where $\bar{f}=f+\Sigma m_{i} p_{i}$, some $p_{i} \in R$. Then if $\bar{t}$ is the trivial section, $((\bar{f}), \bar{t}) \in E(A)$ if and only if degree $p_{i} \geq r$, for all $i$. More generally, $((\bar{f}), \bar{s}) \epsilon$ $E(A)$ if and only if $\bar{f} \in \bar{I}^{r}$, where $\bar{I}$ is the ideal of $\bar{s}$.

Proof. We proceed by induction on the length of $A$, the result being trivial for $A=k$. Given $A^{\prime} \in \underline{C}$ and a basis $m_{1}^{\prime}, \cdots, m_{u+1}^{\prime}$ of $m_{A^{\prime}}$, we may suppose that $A^{\prime} \rightarrow A^{\prime} /\left(m_{u+1}^{\prime}\right)$ is small; rename $\epsilon=m_{u+1}^{\prime}$.

If $\left(\left(f^{\prime}\right), t^{\prime}\right) \in E\left(A^{\prime}\right)$, then by induction we have degree $p_{i} \geq r, i=1,2, \cdots, u$. It remains to show $p_{u+1} \in(X, Y)^{r}$. Suppose $p_{u+1} \in(X, Y)^{d}-(X, Y)^{d+1}$, where $d<r$. Then $\epsilon p_{u+1}=f^{\prime}-\Sigma_{i=1}^{u} m_{i}^{\prime} p_{i} \in\left((X, Y)^{r}, f^{\prime}\right) \subset\left((X, Y)^{d+1}, f^{\prime}\right)$, whence (Lemma 1.4) $p_{u+1} \in\left((X, Y)^{d+1}, f^{\prime}, m_{A^{\prime}}\right)$. Reducing $\bmod m_{A^{\prime}}$ yields $p_{u+1} \epsilon$ $(X, Y)^{d+1}$, a contradiction.

Conversely, suppose degree $p_{i} \geq r$, for all $i$; since the result is true $\bmod (\epsilon)$, by Lemma 1.4 it suffices to show that if $g \in(X, Y)^{p}$ and $\epsilon g \in\left((X, Y)^{p+1}, f^{\prime}\right)$, then $g \in\left((X, Y)^{p+1}, f^{\prime}, m_{A^{\prime}}\right)$. 
If $p \leq r-1$, then $g \in(X, Y)^{p}, \epsilon g \in\left((X, Y)^{p+1}, f^{\prime}\right)=(X, Y)^{p+1}$ already implies that the homogeneous component of degree $p$ in $g$ is killed by $\epsilon$; hence, by flatness, it belongs to $R \otimes m_{A}$.

If $p=r$, let $f_{r}^{\prime}$ be the homogeneous component of degree $r$ of $f^{\prime}$; then $\left((X, Y)^{r+1}, f^{\prime}\right)=\left((X, Y)^{r+1}, f_{r}^{\prime}\right)$. Write $g=g_{r}+b$, where $b$ denotes terms of degree $>r$. Now, $\epsilon g \in\left((X, Y)^{r+1}, f^{\prime}\right)$ implies $\epsilon g_{r}=a f_{r}^{\prime}$; since $f_{r}^{\prime}$ is not a zero-divisor, we have $\alpha=a \epsilon$. But $\epsilon\left(g_{r}-a f_{r}^{\prime}\right)=0$ implies $g_{r}-a f_{r}^{\prime} \in R \otimes m_{A^{\prime}}$, so $g \in\left((X, Y)^{r+1}\right.$, $f^{\prime}, m_{A}$ ).

If $p>r$, assume $g \in(X, Y)^{p}$ and $\epsilon g=A f^{\prime}+b$, where $\operatorname{deg} b>p$. With $f_{r}^{\prime}$ as above, we note it is not a zero-divisor, whence the leading term $A_{p-r}$ of $A$ is of degree $p-r$. If $g_{p}$ is the $p$-component of $g, \epsilon g_{p}=A_{p-r} f_{r}^{\prime}$ implies as above $A_{p-r}=\epsilon a_{p-r}$, so $g_{p}-a_{p-r} f_{r}^{\prime} \in R \otimes m_{A^{\prime}}$. Since we may suppose $a_{p-r} \in(X, Y)^{p-r}$, we have $g-a_{p-r} f^{\prime} \in\left((X, Y)^{p+1}, m_{A}\right)$, as desired. This completes the proof of the first claim.

The final remark follows easily by choosing a $\sigma \in G(A)$ such that $\sigma(\bar{I})=(X, Y)$.

Proposition 1.7. E has a very good deformation theory and is smooth. Further, the tangent space is

$$
\left\{\left.((f+\epsilon g),(X-a \epsilon, Y-b \epsilon))\right|_{g}+a f_{X}+b f_{Y} \in(X, Y)^{r}\right\} .
$$

Proof. The proof follows easily by trivializing the sections involved, using the fact that $H$ and $G$ have very good deformation theories and are smooth, and applying Proposition 1.6. For instance, suppose $\beta=((f+\epsilon g),(X-a \epsilon, Y-b \epsilon))$ is in $E(k[\epsilon])$, with $a, b \in k$. Let $\sigma \in G(k[\epsilon])$ send $X$ to $X+a \epsilon, Y$ to $Y+b \epsilon$. Then $\sigma(\beta)=(\sigma(f+\epsilon g),(X, Y))=\left(\left(f+\epsilon\left(a f_{X}+b f_{Y}+g\right)\right),(X, Y)\right)$, so Proposition 1.6 yields the result.

(1.8) Let $\mu: E \rightarrow H$ be the forgetful morphism which neglects the section. The injectivity of $\mu$ is equivalent to equimultiple sections being uniquely determined by the lifting.

Proposition 1.9. $\mu: E \rightarrow H$ is injective if and only if $f$ is not unitangential, i.e., the leading form $f_{r}$ of $f$ has distinct factors.

Proof. By $[8,1.1 .4]$, it suffices to consider $\mu_{\epsilon}$. If $f$ is unitangential, we may assume after change of coordinates that $f_{r}=Y^{r}$. Then one easily checks that if $\bar{s}$ is defined by $(X-\epsilon, Y)$, then $\left(\left(f+\epsilon f_{X}\right), \bar{t}\right)$ and $\left(\left(f+\epsilon f_{X}\right), \bar{s}\right)$ are in $E(k[\epsilon])$. Thus, $\mu_{\epsilon}$ is not injective.

If $f$ is not unitangential, we can change coordinates and write $f_{r}=Y^{m} g$, where $Y$ does not divide $g$. If $((f),(X-a \epsilon, Y-b \epsilon))$ is in the kernel of $\mu_{\epsilon}$, then $a f_{X}+b f_{Y} \in(X, Y)^{r}$, whence $a Y^{m} g_{X}+b\left(m Y^{m-1} g+Y^{m} g_{Y}\right)=0$. Thus, $Y$ divides $b_{g}$, so $b=a=0$, and $\mu_{\epsilon}$ is injective. 
Proposition 1.10. Equimultiple liftings preserve tangential components. That is, if $(f)=\left(g_{1}\right) \cdots\left(g_{t}\right)$ is a factorization into tangential components, there exists a natural inclusion

$$
E_{(f)} \rightarrow \prod_{i=1}^{t} E_{\left(g_{i}\right)}
$$

with image all tuples with the same equimultiple section.

Proof. Let $((\bar{f}), \bar{s}) \in E_{(f)}(A)$; it suffices to assume $\bar{s}$ is trivial. We show we can write $(\bar{f})$ uniquely as $\left(\bar{g}_{1}\right) \ldots\left(\bar{g}_{t}\right)$, where the $\left(\bar{g}_{i}\right)$ are equimultiple liftings of the $\left(g_{i}\right)$ over the trivial section. We first write $(f)=\left(g_{1}\right)(G)$, and factor $(\bar{f})=$ $\left(\bar{g}_{1}\right)(\bar{G})$. This may be done by an induction on the length of $A$ using a standard formal argument and the key fact that $(X, Y)^{r} \subset\left(g_{1}, G\right)$. The uniqueness follows because $g_{1}$ and $G$ are relatively prime in $R$. Factoring $(\bar{G})$ in this fashion yields the desired morphism. The final remark follows from Proposition 1.6.

Remarks. (1.11) Equimultiple liftings do not, in general, preserve irreducible components (i.e., branches); for instance, $\left(Y\left(Y+X^{3}\right)+\epsilon X^{2}\right)$ is equimultiple along the trivial section, but does not factor $(Y+\epsilon \alpha)\left(Y+X^{3}+\epsilon \beta\right)$. For, that would imply $(\alpha+\beta) Y+\alpha X^{3}=X^{2}$, whence $Y$ divides $X^{2}(1-\alpha X)$, an impossibility.

(1.12) If $f \in k\left[\left[X_{1}, \cdots, X_{n}\right]\right]$ is reduced, then one can define the functor $E$ as above, and all the results in (1.2) through (1.8) carry over verbatim. Note the leading form $f_{r}$ will not in general factor into linear terms. However, we generalize Proposition 1.9 as follows: $\mu: E \rightarrow H$ is not injective if and only if $f_{r}$ is, after a linear change of coordinates, a form in $(n-1)$ variables.

2. Higher order equimultiple functors.

(2.1) We now wish to consider liftings that are not only equimultiple, but equimultiple in the "first infinitesimal neighborhoods" as well. Suppose that $((\bar{f}), \bar{s})$ $\epsilon E(A)$. Let $\bar{\Phi}: B_{\bar{s}} \rightarrow$ Spec $R_{A}$ be the blowing-up of Spec $R_{A}$ along the sheaf of ideals $\bar{I}$ defined by the section $\bar{s}$. Let $\bar{I}_{\bar{s}}=\bar{p}^{-1}(V(\bar{I}))$ denote the exceptional fibre, a divisor in $B_{\bar{s}}$, and let the total transform of $V(\bar{f})$ be $\bar{p}^{-1}(V(\bar{f}))=T(\bar{f})$.

Lemma 2.2. The divisor $T(\bar{f})-r \bar{I}_{\bar{s}}$ is effective and flat over $A$, called the proper transform $P(\bar{f})$.

Proof. If $T=\left(X+m_{1}, Y+m_{2}\right)$, then $B_{\bar{s}}$ is the union of the affines Spec $R_{A}\left[X+m_{1} / Y+m_{2}\right]$ and Spec $R_{A}\left[Y+m_{2} / X+m_{1}\right]$. In the first affine, $\bar{I}_{\bar{s}}$ has local equation $Y+m_{2}$, while $(\bar{f})$ defines $T(\bar{f})$. By Proposition 1.6, $\bar{f} \in \bar{I}^{r}$, whence $\bar{f} /\left(Y+m_{2}\right)^{r} \in R_{A}\left[X+m_{1} / Y+m_{2}\right]$. The result now follows easily.

(2.3) We define the reduced total transform $R(\bar{f})$ to be the divisor $T(\bar{f})$ $(r-1) \bar{I}_{\bar{s}}$. Looking at the closed fibre, $R(f)$ has singularities precisely at $P(f)$ $\cap l_{s}$; the number of such points is $t$, the number of tangent directions of $(f) . P(f)$ 
is the union of the proper transforms of the tangential components. We define

$$
E^{2}(A)=\left\{\left((\bar{f}), \bar{s} ; \bar{s}_{1}, \ldots, \bar{s}_{t}\right) \mid((\bar{f}), \bar{s}) \in E(A) \text { and the } \bar{s}_{i}: \text { Spec } A \rightarrow B_{\bar{s}}\right.
$$

are $A$-sections inducing $\bar{s}\left(\bar{s}=\bar{p} \cdot \bar{s}_{i}\right)$ and

inducing equimultiple sections of $R(\bar{f})$ in $\hat{\mathcal{O}}_{p_{i}}$,

for ${ }_{i}$ a singular point of $\left.R(\bar{f})\right\}$.

The supports of the $s_{i}$ 's are called the first infinitesimal neighborboods of the singularity of $(f)$. As before, there is a natural group action $G \times E^{2} \rightarrow E^{2}$ compatible with the forgetful morphism $E^{2} \rightarrow E$. If $\sigma \in G(A), \alpha \in E^{2}(A)$, then $\sigma(\alpha)$ consists of the lifting $\sigma(\bar{f})$, an equimultiple section $\bar{u}=\bar{s} \cdot \sigma^{-1}$, and equimultiple sections $\bar{s}_{i} \cdot \sigma^{-1}$ in $B_{\bar{u}}$, where we view $\sigma$ also as an isomorphism $B_{\bar{u}} \simeq B_{\bar{s}}$.

Lemma 2.4. Suppose $(f)$ is unitangential. If $\left((\bar{f}), \bar{s}_{;} \bar{s}_{1}\right) \in E^{2}(A)$, then there is a $\sigma \in G(A)$ such that $\sigma\left((\bar{f}), \bar{s} ; \bar{s}_{1}\right)=\left(\sigma(\bar{f}), \bar{t} ; \bar{t}_{1}\right)$, where $\bar{t}$ and $\bar{t}_{1}$ are trivial sections.

Proof. First, there is a $\sigma \in G(A)$ such that $\bar{s} \cdot \sigma^{-1}$ is trivial; so, we may assume $\bar{s}=\bar{t}$. Suppose $f_{r}=Y^{r}$, and let $k[[X, Y, T]] /(X T-Y)$ denote the completion of the local ring of the one point of $B_{t}$ at which $R(f)$ has a singularity. By assumption, $R(\bar{f})$ is equimultiple along some section of $A[[X, T]]$ defined by $(X, T-m)$, for some $m \in m_{A}$, since it lies over the trivial section $\bar{t}$ of $A[[X, Y]]$. But if $\sigma \in G(A)$ sends $X$ to $X$ and $Y$ to $Y+m X$, then $\sigma$ is the desired automorphism.

Proposition 2.5. $E^{2}$ bas a very good deformation theory and is smooth.

Proof. Let $A^{\prime} \rightarrow A$ be small, $A^{\prime \prime} \rightarrow A$ arbitrary, $A^{*}=A^{\prime} \times{ }_{A} A^{\prime \prime}$, and consider

$$
\text { (a): } E^{2}\left(A^{*}\right) \rightarrow E^{2}\left(A^{\prime}\right) \times{ }_{E^{2}(A)} E^{2}\left(A^{\prime \prime}\right) \text {. }
$$

If $\left(\left(f^{\prime}\right), s^{\prime} ; s_{1}^{\prime}, \ldots, s_{t}^{\prime}\right) \in E^{2}\left(A^{\prime}\right),\left(\left(f^{\prime \prime}\right), s^{\prime \prime} ; s_{1}^{\prime \prime}, \ldots, s_{t}^{\prime \prime}\right) \in E^{2}\left(A^{\prime \prime}\right)$ have the same image in $E^{2}(A)$, then (Proposition 1.7) there is a unique $\left(\left(f^{*}\right), s^{*}\right) \in E\left(A^{*}\right)$ lying over the liftings and first equimultiple sections. There are natural isomorphisms $B_{s^{*}} \simeq B_{s^{\prime}} \times_{B} B_{s^{\prime \prime}}$ and $R\left(f^{*}\right) \simeq R\left(f^{\prime}\right) \times_{R(\bar{f})} R\left(f^{\prime \prime}\right)$. Therefore, there are unique sections $s_{i}^{*}$ of $B_{s^{*}}$ lifting the $s_{i}^{\prime}$ and $s_{i}^{\prime \prime}$. A local argument (using Proposition 1.7) shows $\left(\left(f^{*}\right), s^{*} ; s_{1}^{*}, \cdots, s_{t}^{*}\right)$ is in $E^{2}\left(A^{*}\right)$, whence $(\alpha)$ is surjective. The injectivity is similar.

For smoothness, let $A^{\prime} \rightarrow A$ be small, and consider $\left((\bar{f}), \bar{s}_{;} \bar{s}_{1}, \ldots, \bar{s}_{t}\right)$ in $E^{2}(A)$. By Proposition 1.9, we may write $(\bar{f})=\left(\bar{f}_{1}\right) \ldots\left(\bar{f}_{t}\right)$. But Lemma 2.4 implies we may lift each $\left(\left(\bar{f}_{i}\right), \bar{s} ; \bar{s}_{i}\right)$ to $\left(\left(f_{i}^{\prime}\right), s^{\prime} ; s_{i}^{\prime}\right) \in E^{2}\left(A^{\prime}\right)$ (with the same section $s^{\prime}$ for all $i$ ), whence (Proposition 1.10) $\left(\left(f_{1}^{\prime} \cdots f_{t}^{\prime}\right), s^{\prime} ; s_{1}^{\prime}, \cdots, s_{t}^{\prime}\right)$ is in $E^{2}\left(A^{\prime}\right)$ and lifts the original element. 
Remark 2.6. The equimultiple sections in $B_{\bar{s}}$ need not be unique, as seen from the example $\left(\left(Y^{2}+X^{3}+\epsilon X Y\right), \bar{t}\right)$.

(2.7) It is now clear how to define inductively the $n$th equimultiple functor $E^{n}$ on $C$. Suppose $E^{j}$ has been defined for $2 \leq j \leq n-1$, with natural maps $E^{j} \rightarrow$ $E^{j-1}$. For an element $a$ of $E^{j}(A)$, let $a^{*}=\left((\bar{f}), \bar{s}_{j} \bar{s}_{1}, \ldots, \bar{s}_{t}\right)$ denote the image of $\alpha$ in $E^{2}(A)$; also, let $B_{a}$ denote the Spec $A$-scheme obtained by blowing up Spec $R_{A}$ successively along the $A$-sections of $a$. Let $\left(\bar{f}_{i}\right)$ be the ideal of $R(\bar{f}) \subset$ $B_{\bar{s}}$ at the singular point $\bar{p}_{i}$ defined by $\bar{s}_{i}$. There are ordered collections $\alpha_{i}$ of sections in a such that $\left(\left(\bar{f}_{i}\right), a_{i}\right) \in E_{\left(f_{i}\right)}^{j-1}(A)$; this makes sense, because for instance $A$-sections of $B_{\bar{s}} ; \bar{s}_{i}$ inducing $\bar{s}_{i}$ on $B_{\bar{s}}$ yield $A$-sections of the blowingup of Spec $\hat{\Theta}_{\bar{p}_{i}}$ along $\bar{s}_{i}$.

An element of $E^{n}(A)$ is then, by definition, $\left(\alpha ; \bar{u}_{1}, \cdots, \bar{u}_{m}\right)$, where

(a) $a \in E^{n-1}(A)$,

(b) $\bar{u}_{i}$ is an $A$-section of $B_{a}$,

(c) every $\bar{u}_{i}$ lies over a section $\bar{s}_{j}$ of $B_{\bar{s}}$ in $a^{*}$; letting $\bar{u}_{j, 1}, \ldots, \bar{u}_{j, q}$ denote all such sections, we have, for all $j$,

$$
\left(\bar{f}_{j}, a_{j} ; \bar{u}_{j, 1}, \ldots, \bar{u}_{j, q}\right) \in E_{\left(\bar{f}_{j}\right)}^{n-1}(A) .
$$

The supports of the sections in the data of an element of $E^{n}(A)$ are called the (infinitesimal) neigbborboods of $(\bar{f})$. We may further speak of the reduced total transform of $(\bar{f})$ in $B_{a}$; as above, it is a lifting of a reduced divisor supported on the total transform of $(f)$.

Proposition 2.8. $E^{n}$ bas a very good deformation theory for all $n$.

Proof. Using induction on $n$, one proceeds exactly as in Proposition 2.5. The point is that because $E^{n-1}$ satisfies $\left(\mathrm{H}_{4}\right)$, all sections of $E^{n}\left(A^{\prime} \times_{A} A^{\prime \prime}\right)$ are equimultiple and uniquely determined by $E^{n}\left(A^{\prime}\right)$ and $E^{n}\left(A^{\prime \prime}\right)$, except possibly in the last blowing-up. But one then applies Proposition 2.5.

Remarks. (2.9) The usual functorial group action $G \times E^{n} \rightarrow E^{n}$ is defined for every $n$, and commutes with the forgetful maps $E^{n} \rightarrow E^{n-1}$.

(2.10) Presumably all functors $E^{n}$ are smooth. However, Lemma 2.4 does not generalize; we may not be able to trivialize simultaneously all sections of an element of $E^{n}(A)$, even if $f$ is irreducible.

(2.11) Suppose $(f)$ is a curve for which $R(f) \subset B_{s}$ has an ordinary double point at a section $s_{i}$. Then if $((\bar{f}), \bar{s}) \in E(A)$, then there exists a unique $A$-section $\bar{s}_{i}$ of $B_{\bar{s}}$ lifting $s_{i}$ along which $R(\bar{f})$ is equimultiple (by 1.9).

(2.12) If $(f)$ defines an ordinary multiple point (i.e., $f$, has $r$ distinct factors), then one computes that $E^{2} \simeq E$. (Use the previous remark.)

(2.13) Our definition of $E^{n}$ requires that all sections be supported on singular points of $R(f)$ (i.e., points on the proper transform). 
3. The equisingular functor $E S$.

(3.1) It is a well-known fact in the theory of curves that after blowing up Spec $k[[X, Y]]$ and its transforms sufficiently many times, the reduced total transform of $(f)$ has only ordinary double points as singularities.

Theorem 3.2. There exists an integer $N$ such that the natural maps $E^{N+i} \rightarrow$ $E^{N+i-1}$ are bijective, for all $i>0$. For such an $N$, the natural map $E^{N} \rightarrow H$ is injective, unless $f$ is a regular parameter.

Proof. Let $N$ be the smallest positive integer so that in any sequence of the form $\left\{(f),\left(f_{1}\right),\left(f_{2}\right), \ldots\right\}$, where $\left(f_{i+1}\right)$ is the ideal in the complete local ring at a singular point of the reduced total transform of a blowing-up of $\left(f_{i}\right)[10$, p. 512], we have that $\left(f_{j}\right)$ defines an ordinary double point for $j \geq N-1$. It suffices to show $E^{N+1} \rightarrow E^{N}$ is a-bijection. The question being one of existence and uniqueness of equimultiple sections, it suffices to show that for an ordinary double point, $E^{2} \sim E$. But this fact was mentioned in Remark 2.12.

To show $E^{N} \rightarrow H$ is injective, it suffices to check on tangent spaces. Further, by induction on $N$, we have only to check that if $((f), \bar{s}) \in E(k[\epsilon])$ is in the image of $E^{N}(k[\epsilon]) \rightarrow E(k[\epsilon])$, then $\bar{s}$ is the trivial section. The result is obvious by Proposition 1.9 if $f$ is not unitangential.

Thus, suppose $f$ has tangent $Y=0$ and multiplicity $r>1$; since we are only concerned with $(f)$, the Weierstrass preparation theorem allows us to assume $f(X, Y)=Y^{r}+\Sigma_{i=0}^{r-1} d_{i}(X) Y^{i}$, with $d_{i} \in k[[X]]$. After a further coordinate change, $d_{r-1}(X)=0$, so we write $f(X, Y)=Y^{r}+\Sigma_{i \in A} d_{i}(X) Y^{i}$, where $A \subset\{0,1, \cdots, r-2\}$, and $d_{i}(X)$ has leading form $c_{i} X^{e_{i}}$, with $e_{i}>r-i$.

With $((f), \bar{s})$ as above, $\bar{s}$ defined by $(X-a \epsilon, Y)$, we prove $a=0$. Let $\sigma \epsilon$ $G(k[\epsilon])$ send $X$ to $X+a \epsilon, Y$ to $Y$, whence $(\sigma(f), \bar{t})$ is in the image of $E^{N}(k[\epsilon])$. Note

$$
\sigma(f)=Y^{r}+\sum_{i \in A}\left(d_{i}(X)+a \epsilon d_{i}^{\prime}(X)\right) Y^{i}
$$

Since $(\sigma(f), \bar{t})$ is in the image of $E^{2}(k[\epsilon])$, the reduced total transform (with coordinate $\left.T_{1}=Y / X\right)$ in $B_{\bar{t}}$ is given by

$$
\bar{f}_{1}\left(X, T_{1}\right)=X\left(T_{1}^{r}+\sum\left(d_{i}(X)+\operatorname{a\epsilon d}_{i}^{\prime}(X)\right) X^{i-r} T_{1}^{i}\right),
$$

and it is equimultiple along a section of the form $\left(X, T_{1}-b \epsilon\right)$. The multiplicity $q_{1}$ of $f_{1}$ is $\min _{i \in A}\left\{r+1, e_{i}+2 i-r+1\right\}$. We distinguish two cases.

If $q_{1}<r+1$, let $j$ be the largest integer in $A$ such that $e_{j}+2 j-r+1=q_{1}$. Then, by Proposition 1.7,

$$
a \sum d_{i}^{\prime}(X) X^{i-r+1} T_{1}^{i}+b \frac{\partial}{\partial T_{1}}\left(f_{1}\right) \in\left(X, T_{1}\right)^{q},
$$


whence $a c_{j} e_{j}=0$. Since $e_{j}>r-j>0, a=0$, and we are done in this case.

If $q_{1}=r+1$, then, as above, the series

$$
b\left(r X T_{1}^{r-1}+\sum i c_{i} X^{i+e_{i}-r+1} T_{1}^{i-1}\right)+a \sum c_{i} e_{i} X^{e_{i}+i-r} T_{1}^{i}
$$

is in $\left(X, T_{1}\right)^{r+1}$. Since every $i \leq r-2$, the term $b_{r} X T_{1}^{r-1}=0$, whence $b=0$, so that $a=0$ or $e_{i}+2 i-r+1>r+1$, for all $i$. In the second case, $\left(\bar{f}_{1}\right)$ is equimultiple along the trivial section only; since $((\bar{f}), \bar{t} ; \bar{t})$ is in the image of $E^{3}(k[\epsilon])$, we blow up again, and have (with $T_{2}=T_{1} / X$ )

$$
\bar{f}_{2}\left(X, T_{2}\right)=X\left(T_{2}^{r}+\sum\left(d_{i}(X)+a \epsilon d_{i}^{\prime}(X)\right) X^{2 i-2 r} T_{2}^{i}\right)
$$

is equimultiple along a section $\left(X, T_{2}-b \epsilon\right)$. Proceeding as above, we find that either $a=0$ or else $b=0$ and $r+1<e_{i}+3 i-2 r+1$, for all $i \in A$. Repeating this procedure $n$ times, we have either $a=0$ or the reduced total transform is equimultiple along the trivial section and $r+1<e_{i}+(n+1) i-n r+1$, for all $i \in A$. The second case implies $\left.r<\left(e_{i} / n+1\right)\right)+i$; but $A \neq \varnothing$ and $i<r$, so this is a contradiction for $n$ sufficiently large. Therefore, $a=0$, and the theorem is proved.

(3.3) We -can now make the following definition. With $N$ as in Theorem 3.2, we call $E^{N}$ the functor of equisingular liftings, and denote it by $E S$. Since the natural map $E S \rightarrow H$ is an inclusion, we need not mention the sections; although they are part of the data, they are uniquely determined once the lifting is given. Recall (Proposition 1.9) that the functor of equimultiple liftings does not have a good deformation theory-it must be equipped with sections. Note also that in considering equisingular liftings, one need not blow up every section as in each $E^{i}$; one only must blow up sections supported on nonordinary double points, and we can consider any resolution of the singularity $B \rightarrow$ Spec $R$.

We shall frequently say " $(\bar{f})$ is equisingular along $\bar{s}$ " to mean $((T), \bar{s})$ is in the image of $E^{N} \rightarrow E$.

Proposition 3.4. Suppose $(f)=\left(g_{1}\right)\left(g_{2}\right)$. Then there exists a natural injection $E S_{(f)} \rightarrow E S_{\left(g_{1}\right)} \times E S_{\left(g_{2}\right)}$. In particular, equisingular liftings preserve branches.

Proof. Let $(\bar{f}) \in E S_{(f)}(A)$; we claim $(\bar{f})$ factors uniquely as a product $\left(\bar{g}_{1}\right)\left(\bar{g}_{2}\right)$ of equisingular liftings of $g_{1}$ and $g_{2}$. Let $\bar{p}: \bar{B} \rightarrow$ Spec $R_{A}$ be a resolution of the singularity of $(\bar{f})$; that is, the reduced total transform $(\bar{f})$ has only ordinary double points as singularities, all trivially deformed. Let $\bar{T}$ be the ideal sheaf of $\bar{X} \subset \bar{B}$, the proper transform of $(\bar{f})$. It is well known that $\bar{p}_{*}\left(\Theta_{B}\right)=\Gamma\left(\Theta_{\bar{B}}\right)$ $=R_{A} ; R^{1} \bar{p}_{*} \Theta_{\bar{B}}=0$; and $\bar{p}_{*} \overline{\bar{p}} *(\bar{f})=(\bar{f})$. We shall show below that $\bar{p}_{*} \bar{I}=(\bar{f})$; assume it is true.

Reducing $\bmod m_{A}$, we have the resolution $p: B \rightarrow \operatorname{Spec} R$ and the sheaf of ideals $I$ of $X$ on $B$. Since $(f)=\left(g_{1}\right)\left(g_{2}\right)$, we may write $I=I_{1} \cap I_{2}$, where $I_{i}$ is the 
ideal sheaf of the proper transform $X_{i}$ of $\left(g_{i}\right)$. But $X_{1} \cap X_{2}=\varnothing$, so we may write $\bar{I}=\bar{I}_{1} \cap \bar{I}_{2}$ on $\bar{B}$, where $\bar{I}_{i}$ is a uniquely determined lifting of $I_{i}$. Thus,

$$
\bar{p}_{*} \bar{I}=(\bar{f})=\bar{p}_{*}\left(\bar{I}_{1} \cap \bar{I}_{2}\right)=\bar{p}_{*}\left(\bar{I}_{1}\right) \cap \bar{p}_{*}\left(\bar{I}_{2}\right) \text {. }
$$

We claim $\bar{\Gamma}_{*}\left(\bar{I}_{i}\right)$ is a flat $R_{A}$-module, hence a principal ideal; but using the infinitesimal criterion of flatness, one sees that it suffices to show $\bar{p}_{*}\left(\bar{I}_{i}\right)$ is $A$-flat. On $\bar{B}$, we have the exact sequences

$$
\begin{array}{r}
0 \rightarrow \bar{I}_{1} \cap \bar{I}_{2} \rightarrow \Theta_{\bar{B}} \rightarrow \Theta_{\bar{X}_{1}} \oplus \mathcal{O}_{\bar{X}_{2}} \rightarrow 0 \\
\downarrow \quad \| \quad \downarrow \\
0 \longrightarrow \bar{I}_{i} \longrightarrow \Theta_{\bar{B}} \rightarrow \Theta_{\bar{X}_{i}} \longrightarrow 0 .
\end{array}
$$

Taking $\bar{F}_{*}$ yields

$$
\begin{gathered}
0 \rightarrow \Gamma\left(\bar{I}_{1} \cap \bar{I}_{2}\right) \rightarrow \Gamma\left(\Theta_{\bar{B}}\right) \rightarrow \Gamma\left(\Theta_{\bar{x}_{1}}\right) \oplus \Gamma\left(\Theta_{\bar{X}_{2}}\right) \rightarrow R^{1} \bar{p}_{*}\left(\bar{I}_{1} \cap \bar{I}_{2}\right) \rightarrow 0 \\
\downarrow
\end{gathered}
$$

Since the third vertical map is a projection of $\Gamma\left(\Theta_{B}\right)$-modules and hence admits a section, a diagram chase yields an isomorphism $R^{1} \bar{p}_{*}\left(\bar{I}_{1} \cap I_{2}\right) \simeq R^{1} \bar{F}_{*} \bar{I}_{1} \oplus$ $R^{1} \bar{F}_{*} \bar{I}_{2}$. But since the two middle terms of either row are $A$-flat $\left(X_{i}\right.$ is affine), the first module in a row is $A$-flat if and only if the fourth is. But $\Gamma\left(\bar{I}_{1} \cap \bar{I}_{2}\right)=$ $(\bar{f})$ is flat, hence so is $R^{1} \bar{F}_{*}\left(\bar{I}_{1} \cap \bar{I}_{2}\right)$ and each direct summand $R^{1} \bar{T}_{*} \bar{I}_{i}$. Therefore, $\bar{\Phi}_{*} \bar{I}_{i}$ is $A$-flat, hence is a principal ideal $\left(\bar{g}_{i}\right) \subset R_{A}$ lifting $\left(g_{i}\right)$. By construction, $\left(\bar{g}_{i}\right) \in E S_{\left(g_{i}\right)}(A)$. We leave the rest of the details to the reader.

It remains to show $\bar{p}_{*} \bar{I}=(\bar{f})$. We immediately reduce to the case where $\bar{p}: \bar{B} \rightarrow$ Spec $R_{A}$ is one blowing-up along the trivial section. If $\bar{J}$ is the ideal sheaf of the exceptional fibre $\bar{E}$, then we have $\bar{p}^{*}(\bar{f})=\bar{J}^{r} \otimes \bar{I}(r=$ degree of $f)$. We claim

$$
\Gamma\left(\bar{J}^{i} \otimes \bar{I}\right)=\Gamma\left(\bar{J}^{i+1} \otimes \bar{I}\right), \quad i=0,1, \ldots, r-1 .
$$

Due to the exact sequence $0 \rightarrow \bar{J}^{i+1} \otimes \bar{I} \rightarrow \bar{J}^{i} \otimes I \rightarrow J^{i} \otimes T \otimes \Theta_{\bar{E}} \rightarrow 0$, it suffices to show $\Gamma\left(\bar{J}^{i} \otimes \bar{I} \otimes \Theta_{\bar{E}}\right)=0$. However, since $\bar{E}$ is isomorphic to $\mathbf{P}^{1} \times \operatorname{Spec} A$ and $H^{1}\left(\Theta_{\mathrm{P} l}\right)=0$, we have that $\bar{J}^{i} \otimes \bar{I} \otimes \Theta_{\bar{E}}$ is a product deformation of the invertible sheaf $J^{i} \otimes I \otimes \Theta_{E}$ on $E \subset B\left[1\right.$, III. 7]. Now $I \otimes \Theta_{E} \cong \Theta_{E}(-r)$, while $J \otimes \Theta_{E}$ $=\Theta_{E}(1)=$ conormal sheaf of $E$ in $B$; thus, $J^{i} \otimes I \otimes \mathcal{O}_{E} \simeq \mathcal{O}_{E}(-r+i)$. The result now follows because $H^{0}\left(E, \Theta_{E}(-r+i)\right)=0$, for all $i \leq r-1$.

Remarks. (3.5) The above result guarantees that equisingular liftings induce "tangentially stable pairings" of the branches (part of Zariski's definition in [10]). 
(3.6) It is not true that the product of two equisingular liftings is equisingular, unless one knows all sections in all neighborhoods of the liftings are disjoint or coincide. For example, $Y\left(Y+X^{2}+\epsilon X\right)$ is not equisingular. However, we have the

Proposition 3.7. Let $(f)=\left(f_{1}\right) \ldots\left(f_{t}\right)$ be a factorization into tangential components. Then the inclusion $E S_{(f)} \rightarrow \Pi E S_{\left(f_{i}\right)}$ bas as image all products of equisingular liftings with the same equisingular section in Spec $R_{A}, A \in \underline{C}$.

4. The smoothness of ES.

(4.1) Let $(f)$ be a reduced curve, and suppose $p: B \rightarrow$ Spec $R$ resolves the singularity (i.e., $R(f)$ has only nodes as singularities). As usual, we require that all blown-up sections lie on the proper transforms of ( $f$ ) (2.13). Let $E=p^{-1}\left\{m_{R}\right\}$ be the reduced exceptional subscheme; it is a tree of $\mathrm{P}^{1}$ 's. By [EGA, II. 4.6.13], there is an effective divisor $Z$ of $B$, with support $E$, such that $\Theta(-Z)$ is ample for $p$; thus, we may assume $H^{1}\left(T_{B} \otimes Q(-Z)\right)=0$, where we denote by $T_{B}$ the dual of $\Omega_{B / k}^{1}$. Let $L_{Z}$ be the functor on $\underline{C}$ of classes of locally trivial deformations of $Z$ over Spec $k$. From [8], it follows that $L_{Z}$ is versal and smooth (since $H^{2}\left(T_{Z}\right)=0$ ), with tangent space $H^{1}\left(T_{Z}\right)$; further, local triviality is equivalent to formal triviality [8, Proposition 2.1.5]. In this section, we prove the following

Theorem 4.2. There is a natural smooth morphism $\gamma: E S \rightarrow L_{Z}$. In particular, ES is smooth.

(4.3) We start with a description of $L_{E}$. Let $E_{1}, \cdots, E_{s}$ be the components of $E, T_{i}=E_{i} \cap\left(\bigcup_{j \neq i} E_{j}\right)$, and $t_{i}=$ number of elements in $T_{i}$.

Proposition 4.4. There is a natural isomorphism $T_{E} \simeq \bigoplus p_{i_{*}} \Theta_{E_{i}}\left(2-t_{i}\right)$, where $p_{i}: E_{i} \rightarrow E$ is the inclusion. In particular,

$$
\operatorname{dim} H^{1}\left(T_{E}\right)=\sum_{i} \max \left(0, t_{i}-3\right) .
$$

Proof. The natural map $T_{E} \rightarrow p_{i_{*}} T_{E_{i}}$ is easily seen to land in $p_{i_{*}}\left(T_{E_{i}} \otimes I_{i}\right)$, where $I_{i}$ is the sheaf of ideals of the subscheme $T_{i} \subset E_{i}$ (since derivations of $E$ must vanish on the inverse images in $E_{i}$ of the singular points of $E$ ). A local argument shows the induced map $T_{E} \rightarrow \bigoplus p_{i_{*}}\left(T_{E_{i}} \otimes I_{i}\right)$ is an isomorphism; for, if $E$ is given formally at a singular point by $O=k[X, Y]] / X Y$, then the map

$$
\left\{a \frac{\partial}{\partial X}+b \frac{\partial}{\partial Y} \epsilon \mathcal{O} \frac{\partial}{\partial X} \oplus \Theta \frac{\partial}{\partial Y} \mid a Y+b X=0\right\} \rightarrow X k[[X]] \frac{\partial}{\partial X} \oplus Y k[[Y]] \frac{\partial}{\partial Y}
$$

is an isomorphism. But $T_{E_{i}}=\Theta_{E_{i}}(2), I_{i}=\Theta_{E_{i}}\left(-t_{i}\right)$, whence the result.

(4.5) Unfortunately, $T_{Z}$ does not in general admit as simple a description. On the other hand, if $N_{Z}^{0}=\operatorname{Coker}\left(T_{Z} \rightarrow T_{B} \otimes \Theta_{Z}\right)$ (the "locally trivial" normal sheaf, cf. $[8,3.2 .3]$ ), the natural map $N_{Z}^{0} \rightarrow N_{E}^{0}$ is an isomorphism (a formal check). 
On the other hand, an argument as in Proposition 4.4 shows $N_{E}^{0} \simeq \bigoplus p_{i_{*}} N_{E_{i}}$, where $N_{E_{i}}$ is the normal bundle of $E_{i}$ in $B$. Since $H^{0}\left(N_{E_{i}}\right)=0$, there is an exact sequence

$$
0 \rightarrow H^{1}\left(T_{Z}\right) \rightarrow H^{1}\left(T_{B} \otimes \mathcal{O}_{Z}\right) \rightarrow H^{1}\left(N_{E}^{0}\right) \rightarrow 0
$$

(4.6) We want to introduce an auxiliary functor $L$. Note $B$ was obtained from Spec $R$ by a sequence of blowings-up $B=B_{M} \rightarrow B_{M-1} \rightarrow \cdots \rightarrow$ Spec $R$, where $B_{i+1} \rightarrow B_{i}$ is centered at a section $\sigma_{i}$ of the exceptional fibre of $p_{i}: B_{i} \rightarrow$ Spec $R$. Although $B$ is regular and excellent, it is not smooth over $k$ in the usual sense (nor even formally smooth in the sense of Lichtenbaum-Schlessinger [3]), since the local rings have mixed "algebraic" and "continuous" structure over $k$; thus, it is not clear that all deformations of $B$ are locally trivial in the Zariski topology.

If Spec $S=\operatorname{Spec} k[X, Y]$, then it is clear there is a sequence of blowings-up $C=C_{M} \rightarrow C_{M-1} \rightarrow \cdots \rightarrow$ Spec $S$ inducing the resolution $B \rightarrow \operatorname{Spec} R$ via flat base change. Of course, $C$ is now a nonsingular variety over $k$. Denote also by $E$ the exceptional subscheme of $r: C \rightarrow \operatorname{Spec} S$, and by $\sigma_{i}$ the section $C_{i}$ to be blown up. Let $L(A)$ be the set of classes of deformations $\bar{C} \rightarrow \operatorname{Spec} A$ which may be obtained by a sequence of blowings-up $\bar{C} \rightarrow \bar{C}_{M-1} \rightarrow \cdots \rightarrow$ Spec $S_{A}$, where $\bar{C}_{i+1} \rightarrow \bar{C}_{i}$ is centered at a normally flat $A$-section $\bar{\sigma}_{i}$ of the exceptional subscheme of $\bar{C}_{i} \rightarrow \operatorname{Spec} S_{A}$ lifting $\sigma_{i}$ and inducing $\bar{\sigma}_{i-1}$.

Lemma 4.7. $L$ is versal and smooth, and is the functor of those deformation classes of $C$ to which $E$ admits a locally trivial lifting.

Proof. Since $T_{C}$ is coherent, $H^{1}\left(T_{C}\right)=R^{1} r_{*} T_{C}$ is a finite $k[X, Y]$-module ( $r$ is proper); since it is supported on $V(X, Y), H^{1}\left(T_{C}\right)$ is finite over $k$, whence the functor of deformation classes of $C$ is versal [6]. Since $L$ is contained in this functor, it suffices to show $L$ satisfies $\left(\mathrm{H}_{1}\right)$. One can do this once we show that if $A^{\prime} \rightarrow A$ is small, two Spec $A^{\prime}$-morphisms $C^{\prime} \rightarrow \operatorname{Spec} S_{A^{\prime}}$, inducing the same map over Spec $S_{A}$, differ by an automorphism of Spec $S_{A^{\prime}}$. But this follows

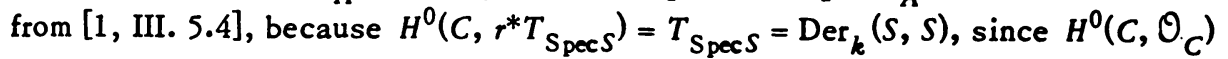
$=S$. The smoothness of $L$ is obvious.

If $f: X \rightarrow Y$ is a blowing-up of a (closed) point $P$ of a nonsingular variety $Y$ of dimension $\geq 2$, then one establishes easily the exact sequence

$$
0 \rightarrow f_{*} T_{X} \rightarrow T_{Y} \rightarrow N_{P} \rightarrow 0,
$$

where $N_{P}$ is the normal sheaf of $P$ in $Y$. There is a morphism of the functor of deformation classes of $Y$ and liftings of $P$ into the functor of deformation classes of $X$; the exact sequence

$$
H^{0}\left(N_{P}\right) \rightarrow H^{1}\left(T_{X}\right) \rightarrow H^{1}\left(T_{Y}\right) \rightarrow 0
$$


shows the morphism is surjective on the tangent spaces, whence the usual argument shows it is surjective. We conclude that every deformation class $[\bar{C}]$ of $C$ is obtained from Spec $S_{A}$ by a sequence of blowings-up.

Any element of $L(A)$ certainly induces a locally trivial deformation of $E$. For the converse, one uses induction and reduces to proving the following: if $(g) \subset$ $k[[X, Y]]$ is $(X)$ or $(X Y),(\bar{g}) \subset R_{A}$ a lifting, and $\bar{s}$ an $A$-section of $R_{A}$, then the total transform $T(\bar{g}) \subset B_{\bar{s}}(2.1)$ is a locally trivial deformation of $T(g)$ if and only if $((\bar{g}), \bar{s}) \in E_{(g)}(A)$. The proof is an easy computation.

(4.8) From obstruction theory and the map $T_{C} \rightarrow T_{C} \otimes \Theta_{E} \rightarrow N_{E}^{0}$, one has that

$$
L(k[\epsilon])=\operatorname{Ker}\left(H^{1}\left(T_{C}\right) \rightarrow H^{1}\left(N_{E}^{0}\right)\right) .
$$

On the other hand, $Z$ was chosen so that $H^{1}\left(T_{C}\right) \simeq H^{1}\left(T_{C} \otimes \Theta_{Z}\right)$, whence, as in (4.5), we have $L(k[\epsilon])=H^{1}\left(T_{Z}\right)$. In fact, we have

Proposition 4.9. There is a natural isomorphism $\beta: L \approx L_{Z} \cdot$

Proof. Since $L$ is smooth and $\beta_{\epsilon}$ is surjective (4.8), it follows that $\beta$ is surjective $[8,1.1 .4]$.

To show $\beta$ is injective, suppose $C_{i}^{\prime} \rightarrow$ Spec $A^{\prime}$ induce elements of $L\left(A^{\prime}\right)$ with the same image in $L_{Z}\left(A^{\prime}\right)$; we may suppose they are isomorphic over $\operatorname{Spec} A$, where $A^{\prime} \rightarrow A$ is small. Let $Z_{i}^{\prime} \subset C_{i}^{\prime}$ be the induced subschemes, and consider the diagram

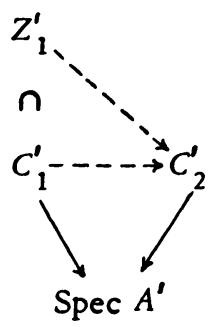

The obstruction to lifting the isomorphism over $A$ to an $A^{\prime}$-homomorphism $C_{1}^{\prime} \rightarrow$ $C_{2}^{\prime}$ lies in $H^{1}\left(T_{C}\right)$ [1, III. 5.4], while the obstruction to lifting to a map $Z_{1}^{\prime} \rightarrow$ $C_{2}^{\prime}$ is easily seen to lie in $H^{1}\left(T_{C} \otimes \Theta_{Z}\right)$. But by assumption, $H^{1}\left(T_{C}\right) \simeq$ $H^{1}\left(T_{C} \otimes \Theta_{Z}\right)$; thus, if $Z_{1}^{\prime} \propto Z_{2}^{\prime}$, the obstruction vanishes, so $C_{1}^{\prime}$ and $C_{2}^{\prime}$ are necessarily $A^{\prime}$-isomorphic.

Proof of Theorem 4.2. Suppose $Z=\Sigma r_{i} E_{i}$. If $(\bar{f}) \in E S(A)$, one blows up successively the equisingular sections of $(\bar{f})$ and its transforms to get a map $\bar{p}$ : $\bar{B} \rightarrow$ Spec $R_{A}$; such a process is well defined, since all sections are unique ((2.13) and Theorem 3.2). Let $\gamma_{A}((\bar{f}))$ be the deformation class of $\bar{Z}=\Sigma r_{i} \bar{E}_{i}$, where $\bar{E}_{i}$ is the unique lifting of $E_{i}$ to $\bar{B}$. As in (4.6), $\bar{P}$ is induced by $\bar{C} \rightarrow$ Spec $S_{A}$. 
If $\left[Z^{\prime}\right] \in L_{Z}\left(A^{\prime}\right)$ lifts $\bar{Z}$; where $A^{\prime} \rightarrow A$ is small, then by Proposition 4.9, there is a $C^{\prime} \rightarrow \operatorname{Spec} S_{A^{\prime}}$ lifting $\bar{C}$ and containing $Z^{\prime}$; suppose $C^{\prime}$ induces $p^{\prime}$ : $B^{\prime} \rightarrow$ Spec $R_{A^{\prime}}$. The total transform $T(\bar{f}) \subset \bar{B}$ is defined by the free sheaf of $\Theta_{B^{-}}$ modules, $\bar{p}^{*}(\bar{f})$, which consists of the product of the ideal sheaves of $n_{i} \bar{E}_{i}$ (some $n_{i}$ ) and of the proper transform of $\bar{f}$. Having lifted the $\bar{E}_{i}$ to $E_{i}^{\prime} \subset B^{\prime}$, one can easily lift $T(\bar{f})$ in a locally trivial fashion to a relative $A^{\prime}$-divisor on $B^{\prime}$, defined by the sheaf of $\Theta_{B^{\prime}}$-ideals $K^{\prime}$. But $K^{\prime}$ is a free $\Theta_{B^{\prime}}$-module, since it lifts the free $\Theta_{B}$-module $p^{*}(f)$ and since $H^{1}\left(B, \Theta_{B}\right)=0\left[1\right.$, III. 7.1]. Thus, $p_{*}^{\prime}\left(K^{\prime}\right) \subset R_{A^{\prime}}$ is a free $R_{A^{\prime}}$-module, hence is a principal ideal $\left(f^{\prime}\right)$ lifting $(\bar{f})$. Further, the natural map $p^{\prime} p_{*}^{\prime} K^{\prime} \rightarrow K^{\prime}$ of free $\Theta_{B^{\prime}}$-modules is necessarily an isomorphism.

We must show $\left(f^{\prime}\right) \in E S\left(A^{\prime}\right)$. Let $E_{1} \subset E$ correspond to the blowing-up of the origin in Spec $R$; then the multiplicity of $E_{1}$ in $p^{*}(f)$ is $r$, the multiplicity of $(f)$. By construction, $f^{\prime}$ vanishes $r$ times on $E_{1}^{\prime}$; equivalently (Proposition 1.6), $\left(f^{\prime}\right)$ is equimultiple along the section defined by $\Gamma\left(\Theta_{B^{\prime}}\right)=R_{A^{\prime}} \rightarrow \Gamma\left(\Theta_{E_{1}^{\prime}}\right) \simeq A^{\prime}$. Next, let $p_{1}^{\prime}: B_{1}^{\prime} \rightarrow$ Spec $R_{A^{\prime}}$, be the blowing-up of this section; then $p^{\prime}$ factors $B^{\prime} \stackrel{q^{\prime}}{\rightarrow} B_{1}^{\prime} \stackrel{p_{1}^{\prime}}{\rightarrow}$ Spec $R_{A^{\prime}}$. If $E_{2} \subset E$ comes from blowing up a point of $B_{1}$, then the morphisms $\Theta_{B_{1}^{\prime}} \simeq q_{*}^{\prime} \Theta_{B^{\prime}} \rightarrow q_{*}^{\prime} \Theta_{E_{2}^{\prime}} \simeq A^{\prime}$ gives rise to the section of $B_{1}^{\prime}$ to be blown up. Thus, equimultiplicity of the transform of $\left(f^{\prime}\right)$ along this section is equivalent to $f^{\prime}$ vanishing sufficiently many times on $E_{2}^{\prime} \subset B^{\prime}$. But this is guaranteed by construction. In this fashion, we find $\left(f^{\prime}\right) \in E S\left(A^{\prime}\right)$, so $\gamma$ is smooth.

Remarks. (4.10) The proof shows that if $Z$ is any divisor for which $H^{1}\left(T_{C}\right)$ $\simeq H^{1}\left(T_{C} \otimes \Theta_{Z}\right)$ ("the deformations of $C$ are determined by those of $Z$ "), then $L \simeq L_{Z}$.

(4.11) One can show that $E S \rightarrow L_{E}$ is smooth; $L_{E}$ is pro-representable and has tangent space described by Proposition 4.4 .

(4.12) For a direct proof of the smoothness of ES, see [9, p. 134].

5. The functor $E S^{\prime}$.

(5.1) Let $p: B \rightarrow \operatorname{Spec} R$ be a minimum resolution of the singularity of $(f)$; that is, one blows up only nonnodes of the reduced total transforms.

Lemma 5.2. If $(\bar{f}) \in E S(A)$ is equisingular along the trivial section in all neighborboods of $B \rightarrow \operatorname{Spec} R$ except at nodes, then it is automatically equisingular along the trivial section at nodes of the exceptional fibre.

Proof. We may restrict ourselves to the following case. Suppose $\mathcal{O}_{i}$ is the local ring of a point $P$ in $B_{i},(\bar{f})$ is equisingular along the trivial section in $\mathcal{O}_{i}$ $\otimes A$, while $Q \in B_{i+1}$ (the blowing-up of $P$ ) is a point not on the proper transform of $(f)$, but at which $R(f)$ has a node. Let $\theta_{i+1}$ be the local ring of $Q$. We may assume $(\bar{f})=(Y)(\bar{g})$ in $\Theta_{i} \otimes A$, where $Y=0$ is the local equation of an exceptional line (as all previous blown-up sections are trivial). Therefore, the reduced 
total transform of $(\bar{f})$ in $\Theta_{i+1} \otimes A$ is given by $(X T)$ (where $Y=X T$ ), hence is equisingular along the trivial section.

Convention (5.3). We shall say that those $(\bar{f})$ as in Lemma 5.2 are equisingular via trivial sections, recalling that this does not apply to nodes of the proper transforms. This is not to be confused with " $(\bar{f})$ equisingular along the trivial section" (see (3.3)).

(5.4) Consider the subfunctor of ES defined by $E S^{\prime}(A)=\{(\bar{f}) \in E S(A) \mid \sigma(\bar{f})$ is equisingular via trivial sections, some $\sigma \in G(A)\}$. Thus, $E S^{\prime}$ consists of the "easiest" equisingular liftings; one does not worry about equisingular sections.

Proposition 5.5. ES' bas a very good deformation theory and is smooth.

Proof. If $(\bar{f})$ and $\sigma(\bar{f})$ are equisingular via trivial sections, then we may view $\sigma$ as an element of $G^{\prime}(A)$, the group of infinitesimal Spec $A$-automorphisms of $B \times \operatorname{Spec} A$. The result follows directly once we show $G^{\prime}$ is smooth. For this, one imitates the proof of $[8,1.3 .1]$, in showing that one can "exponentiate in the Lie algebra $G^{\prime}(k[\epsilon])$ ". In particular, if $d \in G^{\prime}(k[\epsilon]), m \in m_{A}$, one can define $e^{m d} \epsilon$ $G^{\prime}(A)$, and prove that every element of $G^{\prime}(A)$ is a composite of such Spec $A$-automorphisms.

Remark 5.6. One could have started by defining $E S^{\prime}$ as a subfunctor of $H$, and proving directly as above that $E S^{\prime}$ has a very good deformation theory and is smooth; one can therefore avoid Theorems 3.2 and 4.2 for this simpler functor. However, as we shall see below, $E S^{\prime}$ is generally smaller than ES. Denote henceforth by $L$ the functor $L_{Z}$, where $Z$ is as in (4.1).

Theorem 5.7. $E S^{\prime}$ is the kernel of the smooth morphism $\gamma: E S \rightarrow L$. That is, $E S^{\prime}(A)=\left\{(\bar{f}) \in E S(A) \mid \gamma_{A}((\bar{f}))\right.$ is the trivial element of $\left.L(A)\right\}$.

Proof. If $(\bar{f})$ is equisingular via trivial sections, then the induced deformation $\bar{B}$ is trivial, whence so is the element of $L(A)$; therefore, $\operatorname{ES}^{\prime}(A)$ is in the kemel of $E S(A) \rightarrow L(A)$. Conversely, if $(\bar{f}) \in E S(A)$ induces a trivial element of $L(A)$, then by Proposition 4.9 it induces a trivial deformation of $B$. So, if $\bar{B}_{M} \rightarrow$ $\bar{B}_{M-1} \rightarrow \cdots \rightarrow \operatorname{Spec} R_{A}$ is a resolution attached to $(\bar{f})$, then after automorphism $\sigma \in G(A)$, it becomes $B_{M} \times \operatorname{Spec} A \rightarrow \cdots \rightarrow \operatorname{Spec} R \times \operatorname{Spec} A$ (cf. Lemma 4.7). Therefore, $\sigma(\bar{f})$ is equisingular via trivial sections, whence $(\bar{f}) \in E S^{\prime}(A)$.

Corollary 5.8. $E S^{\prime}=E S$ if and only if $L$ is trivial.

6. The tangent space of $E S$.

Proposition 6.1. The tangent space of ES, $I=\{g \in k[[X, Y]] \mid(f+\epsilon g) \epsilon$ $\operatorname{ES}(k[\epsilon])\}$, is an ideal. 
Proof. $I$ is certainly a $k$-module because ES has a good deformation theory. Thus, we must show $g \in I$ implies $a g \in I$, any $a \in R=k[[X, Y]]$. Since, for $\sigma \in G(k[\epsilon])$, there are $p, q \in R$ such that $\sigma(f+\epsilon g)=\left(f+\epsilon\left(g+p f_{X}+q f_{Y}\right)\right)$, it follows that $I$ contains the ideal $\left(f, f_{X}, f_{Y}\right)$; further, we may suppose $(f+\epsilon g)$ is equisingular along the trivial section, and prove $(f+\epsilon \alpha g) \in E S(k[\epsilon])$.

We use induction on $M$, the number of blowings-up needed to resolve the singularity of $(f)$. For $M=1,(f)$ is an ordinary multiple point, and $I=\left(f, f_{X}, f_{Y}\right.$, $\left.(X, Y)^{\eta}\right)$, since in this case equimultiplicity is the same as equisingularity. If $(f)=\left(f_{1}\right) \cdots\left(f_{t}\right)$ is a product of tangential components, then $(f+\epsilon g)=\Pi\left(f_{i}+\epsilon g_{i}\right)$ (Proposition 1.10), whence $(f+\epsilon a g)=\Pi\left(f_{i}+\epsilon a g_{i}\right)$; thus, it suffices to prove the result when $(f)$ is unitangential. But after another automorph ism (Lemma 2.4), we may assume $(f+\epsilon g)$ and its first transform are equisingular along the trivial section. A direct computation now shows that $(f+\epsilon a g)$ and its first transform are equimultiple along the trivial section; but induction in the first neighborhood implies $(f+\epsilon a g) \in E S(k[\epsilon])$.

(6.2) To get a better hold of $I$, we let $B \rightarrow$ Spec $R$ be a minimal resolution of (f) (5.1). Let $\left\{\Theta_{a}\right\}_{a \in T}$ be the set of $\mathcal{O}_{0}=R$ and all local rings of the $B_{i}$ in the resolution at which the reduced total transform has a singularity. Thus, there are $t$ such local rings $\theta_{1}, \ldots, \theta_{t}$ in $B_{1}$, where $t$ is the number of tangential components of $(f)$. Let $S \subset T$ be the index set corresponding to the nonnodes. Let $m_{a} \subset \Theta_{a}$ be the maximal ideal, and let $v_{a}$ be the $m_{a}$-adic valuation on $\vartheta_{a}$. Of course, $R \subset \Theta_{a}$ is birational.

Proposition 6.3. $(f+\epsilon g) \in E S^{\prime}(k[\epsilon])$ if and only if $g$ is in the ideal $l^{\prime}=\left(f, f_{X}\right.$, $\left.f_{Y}, \bigcap_{a \in S} m_{a}^{v_{\alpha}(f)}\right)$. In particular, $I^{\prime} \subset l$.

Proof. If $(f+\epsilon g) \in E S^{\prime}(k[\epsilon])$, there is a $\sigma \in G(k[\epsilon])$ such that $\sigma(f+\epsilon g)$ is equisingular via trivial sections. Since $\left(f, f_{X}, f_{Y}\right) \subset I^{\prime}$, it suffices to assume $(f+\epsilon g)$ is equisingular via trivial sections. The result then follows by definition and Proposition 1.6.

Conversely, suppose $g \in I^{\prime}$; writing $g=a f+b f_{X}+c f_{Y}+g_{1}$, where $g_{1} \epsilon$ $\bigcap_{a \in S} m_{a}^{v_{a}(f)}$, one has $(f+\epsilon g) \in E S^{\prime}(k[\epsilon])$ if and only if $\left(f+\epsilon g_{1}\right) \in E S^{\prime}(k[\epsilon])$. Thus, it suffices to show $g \in \bigcap_{a \in S} m_{a}^{v_{a}(f)}$ implies $(f+\epsilon g)$ is equisingular via trivial sections. If $M=1,(f)$ is an ordinary multiple point, and this case is clear. In the general case, certainly $(f+\epsilon g)$ is equimultiple along the trivial section. If $Y+$ $\beta_{i} X, i=1, \cdots, t$, are linear forms defining the tangent directions of $(f)$, then

$$
\mathcal{O}_{i}=(k[[X, Y]][T] / Y-T X)_{\left(X, T+\beta_{i}\right)}
$$

But the reduced total transform of $(f+\epsilon g)$ in $\mathcal{O}_{i}[\epsilon]$ is given by $(f+\epsilon g) / X^{r-1}=$ $\bar{f}_{i}+\epsilon \bar{g}_{i}$. For any $\mathcal{O}_{a}(a \in S)$ dominating $\mathcal{O}_{i}$, we have $v_{a}(f)=v_{a}\left(\bar{f}_{i}\right)+v_{a}\left(X^{r-1}\right)$ 
and $v_{a}(g)=v_{a}\left(\bar{g}_{i}\right)+v_{\alpha}\left(X^{r-1}\right)$. Thus, $v_{\alpha}(g) \geq v_{\alpha}(f)$ (i.e., $g \in m_{a}^{v_{\alpha}(f)}$ ) if and only if $v_{a}\left(\bar{g}_{i}\right) \geq v_{a}\left(\bar{f}_{i}\right)$. Since taking completions does not change the values of $v_{a}$, the inductive assumption is fulfilled for $\left(\bar{f}_{i}+\epsilon \bar{g}_{i}\right)$, unless $\left(\bar{f}_{i}\right)$ defines an ordinary double point. Since this case is covered by Remark 2.11 , we are done.

Remarks. (6.4) Of course, $I \subset\left(f, f_{X}, f_{Y},(X, Y)\right)$, since equisingular liftings are equimultiple.

(6.5) If $B_{i}$ is some blowing-up in a minimal resolution of $(f)$, and if the corresponding functor $L$ of $B_{i}(4.6)$ is trivial, then one easily shows

$$
I \subset\left(f, f_{X}, f_{Y}, \bigcap_{a \in S_{i}} m_{a}^{v a^{(f)}}\right),
$$

where $S_{i} \subset S$ corresponds to the local rings $\Theta_{a}$ contained in the local rings of $B_{i}$.

Proposition 6.6. In case $f(X, Y)=Y^{p}+X^{q}(p \leq q)$, then $I=I^{\prime}$ is the ideal generated by $X^{q-1}, Y^{p-1}$, and the monomials $X^{i} Y^{j}$, where $p i+q j \geq p q$. In particular, $(f)$ bas nontrivial equisingular liftings if and only if $p=3, q>5$ or $p>3$.

Proof. We proceed by induction on $p q$, the case $p q=1$ being trivial; also, (2.12) proves the result for $p=q$.

Suppose $p<q$, and $\left(Y^{p}+X^{q}+\epsilon \Sigma a_{i j} X^{i} Y^{j}\right) \epsilon E S(k[\epsilon])$; we may as well suppose $i \leq q-2, j \leq p-2$, and the lifting is equisingular along the trivial section. Letting $Y=T X$, the reduced total transform is given by

$$
X\left(T^{p}+X^{q-p}+\epsilon \sum a_{i j} X^{i+j-p} T^{j}\right) .
$$

By Proposition 3.4, $\left(T^{p}+X^{q-p}+\epsilon \Sigma a_{i j} X^{i+j-p} T^{j}\right)$ is equisingular; since $p(q-p)<p q$, we may use induction. An easy computation shows $p i+q j \geq p q$, for all $i, j$ such that $a_{i j} \neq 0$.

Conversely, it suffices to show $\left(Y^{p}+X^{q}+\epsilon X^{i} Y^{j}\right) \epsilon E S(k[\epsilon])$, for $p i+q j \geq p q$. This may be done using Zariski's discriminant criterion for the formal family over $k[[t]]$ defined by $Y^{p}+X^{q}+t X^{i} Y^{j}[10$, Theorem 7]. However, we show directly (by induction) that $\left(Y^{p}+X^{q}+\epsilon X^{i} Y^{j}\right)$ is equisingular via trivial sections if $p i+$ $q j \geq p q$. Since $i+j \geq p$, the lifting is equimultiple along the trivial section; letting $Y=T X$, induction shows the proper transform $\left(T^{p}+X^{q-p}+\epsilon X^{i+j-p} T^{j}\right)$ is equisingular via trivial sections. To show this for $X\left(T^{p}+X^{q-p}+\epsilon X^{i+j-p} T^{j}\right)$, we must prove that the equisingular sections of each component are compatible in all neighborhoods. However, since these sections are almost all trivial, one need only consider the following case: there is an ordinary double point of the reduced total transform of $T^{p}+X^{q-p}$ at which the transform of $X\left(T^{p}+X^{q-p}\right)$ does not have a node. An elementary argument shows this occurs only if $q=p+1$. But a direct computation then shows that $p i+q j \geq p q$ implies $X\left(T^{p}+X+\epsilon X^{i+j-p} T^{j}\right)$ is equisingular via trivial sections. This completes the proof. 
Corollary 6.7. In case $f(X, Y)=Y^{p}+X^{q}$, then $E S^{\prime}=E S$.

Proof. $E S^{\prime} \rightarrow E S$ is surjective on the tangent spaces, and $E S^{\prime}$ is smooth, whence $E S^{\prime} \rightarrow E S$ is surjective.

Example 6.8. Consider the reduced curve defined by $f(X, Y)=\left(X^{4}-Y^{4}\right)^{2}-$ $X^{10}$. This curve has four tangential components, each of which consists of two nonsingular branches with a first-order contact (i.e., the branches become transversal after one blowing-up). If $B_{1} \rightarrow$ Spec $R$ is one blowing-up of the origin, the singularities of $R(f)$ are four ordinary triple points. Thus, $(f+\epsilon g)$ is equisingular via trivial sections precisely when $g$ is of the form $g_{8}+g_{9}+g^{\prime}$, where $g_{8}=$ $c\left(X^{4}-Y^{4}\right)^{2}(c \in k), g_{9} \in\left(X^{2}, X Y, Y^{2}\right) \cdot\left(f_{X}, f_{Y}\right)$, and $g^{\prime} \in(X, Y)^{10}$ (verification is straightforward). Therefore, $I^{\prime}=\left(f, f_{X}, f_{Y},(X, Y)^{10}\right)$.

However, one computes that

$$
(f+\epsilon g)=\left(\left(X^{4}-Y^{4}\right)^{2}-X^{10}+\epsilon X\left(X^{4}-Y^{4}\right)\left(X^{3}+X^{2} Y+X Y^{2}+Y^{3}\right)\right)
$$

is equimultiple along the trivial section, and $R(f+\epsilon g)$ is equimultiple along four sections of $B_{1}[\epsilon]$, only three of which are trivial. Further, $g \notin I^{\prime}$ (essentially because $\left.X^{2} Y^{2}\left(X^{4}-Y^{4}\right) \notin I^{\prime}\right)$, whence $I^{\prime}<I$. In fact,

$$
I=\left(I^{\prime}, X^{2} Y^{2}\left(X^{4}-Y^{4}\right)\right)
$$

This extra equisingular lifting arises from the one-dimensional vector space $L_{E}(k[\epsilon])$ (the exceptional fibre $E$ of a minimal resolution consists of four lines intersecting a given line in four points) via the smooth morphism $E S \rightarrow L$

(Theorem 5.7) and $L \rightarrow L_{E}$ (in this case a bijection).

Remark 6.9. Via (5.7) and (6.3), a thorough knowledge of $E S(k[\epsilon])$ requires knowledge of $H^{1}\left(T_{Z}\right)$, where $Z$ is a certain nonreduced curve. We hope to return to this question in a future paper.

7. Equisingular deformation classes.

(7.1) The notions of equimultiplicity and equisingularity may be considered in terms of the local ring of the singularity, and not just in terms of the embedding in Spec $R$. As in (1.2), we may speak of the functor $D$ of classes of deformations of $P=R /(f)$; assume $P$ is not regular.

If $\bar{P}$ is a deformation of $P$ to $A$, and if $\bar{s}: \bar{P} \rightarrow A$ is a normally flat $A$-section inducing the canonical map $s: P \rightarrow k$, then we say $(\bar{P}, \bar{s})$ is an equimultiple deformation of $(P, s)$. Two such pairs $\left(\bar{P}_{1}, \bar{s}_{1}\right)$ and $\left(\bar{P}_{2}, \bar{s}_{2}\right)$ are said to be equivalent if there is an $A$-isomorphism $\phi: \bar{P}_{1} \rightarrow \bar{P}_{2}$, inducing the identity over $k$, such that $\bar{s}_{2} \cdot \phi=\bar{s}_{1}$. We then define the functor of equimultiple deformation classes plus sections by

$$
\bar{E}(A)=\text { set of equivalence classes of }(\bar{P}, \bar{s}) \text {. }
$$


Proposition 7.2. $\bar{E}$ is the quotient functor of $E$ by $G$, hence is versal and smooth. The natural morphism $\bar{E} \rightarrow D$ is injective if and only if $(f)$ is not unitangential.

Proof. The group action was defined in 1.5; that $\bar{E}$ is the quotient is straightforward, so one applies [8, Proposition 1.1.6]. The finite dimensionality of $\bar{E}(k[\epsilon])$ follows from that of $D(k[\epsilon])$, since there is a two-dimensional space of $k[\epsilon]$-sections of $R[\epsilon]$ inducing the trivial section over $k$. The second statement follows easily from Proposition 1.9.

(7.3) One can define higher order functors $\bar{E}^{n}$ of equimultiple deformation classes plus sections; again, they are the quotients of the actions $G \times E^{n} \rightarrow E^{n}$ and are versal.

In particular, for $N$ large enough, $\bar{E}^{N} \simeq \bar{E}^{N+1} \simeq \ldots$ is the quotient of $E S$ by $G$, the functor $\overline{E S}$ of equisingular deformation classes (plus sections). By Theorem 3.2,ES $\hookrightarrow H$ is injective, hence so is the map of $G$-quotients $\overline{E S} \hookrightarrow D$, and we can again neglect the (uniquely determined) sections. The fundamental morphism $E S \rightarrow L$ factors through $\overline{E S}$, whence there is a smooth morphism $\overline{E S}$ $\rightarrow L$. We also have a smooth versal subfunctor $\overline{E S}^{\prime}$ of $\overline{E S}$. Gathering up our previous results, we have

Theorem 7.4. The functor $\overline{E S}$ of equisingular deformation classes is a versal and smooth subfunctor of the deformation functor $D$ of the singularity. The tangent space is given by $I /\left(f, f_{X}, f_{Y}\right)$. There is a smooth morphism $\overline{E S} \rightarrow L$, with "kernel" $\overline{E S}$ ', where $L$ is the locally trivial deformation functor of a projective curve. If $(f)=\left(f_{1}\right) \ldots\left(f_{s}\right)$ is a factorization into irreducible factors, there is a natural map

$$
\overline{E S}_{(f)} \rightarrow \prod \overline{E S}_{\left(f_{i}\right)}
$$

8. Formal versal equisingular deformations.

(8.1) An important property of the deformation functor of a complete intersection with isolated singularity is the fact that a formal versal deformation may be written down from the tangent space [7]. In particular, if $(f)$ defines a (reduced) algebroid curve, and if $g_{1}, \cdots, g_{m} \in k[[X, Y]]$ have residues forming a basis of $k[[X, Y]] /\left(f, f_{X}, f_{Y}\right)$, then the homomorphism

$$
k\left[\left[t_{1}, \cdots, t_{m}\right]\right] \rightarrow k\left[\left[X, Y, t_{1}, \cdots, t_{m}\right]\right] /\left(f+\sum t_{i} g_{i}\right)
$$

is a formal versal deformation of the singularity.

It would be nice to be able to write down a formal versal equisingular deformation, and not just the generic first-order one (given by the tangent space). The next result indicates that $E S^{\prime}$ is the "maximum" subfunctor with the property that a versal family involves only linear terms in the $t_{i}$ 's. 
Theorem 8.2. Let $(f) \subset R$ define a reduced curve. Then the following are equivalent:

(a) $E S^{\prime}=E S$;

(a') $I^{\prime}=I$

(b) $L$ is trivial for a minimal resolution of $(f)$;

(c) there are elements $g_{1}, \cdots, g_{s} \in I$ such that the formal family

$$
k\left[\left[t_{1}, \cdots, t_{s}\right]\right] \rightarrow k\left[\left[X, Y, t_{1}, \cdots, t_{s}\right]\right] /\left(f+\sum_{i=1}^{s} t_{i} g_{i}\right)
$$

defines a versal deformation for $\overline{E S}$.

Proof. The equivalence of (a), ( $\left.a^{\prime}\right)$, and (b) has already been observed.

Suppose then $E S^{\prime}=E S$, and let $g_{1}, \cdots, g_{s} \in I$ induce a basis of $\overline{E S}(k[\epsilon])$; we may also suppose that $\left(f+\epsilon g_{i}\right)$ is equisingular via trivial sections, for all $i$. Let $C=k\left[\left[t_{1}, \cdots, t_{s}\right]\right]$, and define a morphism $\phi: b_{C} \rightarrow \overline{E S}$ by associating to each local map $a: C \rightarrow A$ the deformation class defined by $\left(f+\Sigma \alpha\left(t_{i}\right) g_{i}\right)$. An easy induction on the number of blowings-up needed to resolve the singularity shows that this lifting is equisingular via trivial sections, whence $\phi$ is well defined. Since $\phi_{\epsilon}$ is a bijection, $\phi$ will be versal once we show it is smooth. One easily reduces to the proof of the following: if $A^{\prime} \rightarrow A$ is small of kernel $(\eta)$, and if $\left(f+\Sigma m_{i}^{\prime} g_{i}+\eta g\right) \in E S^{\prime}\left(A^{\prime}\right)$ is equisingular via trivial sections, then so is $(f+\epsilon g)$ in $E S^{\prime}(k[\epsilon])$. Again, the straightforward proof is by induction on the number of blowings-up. Thus, (a) implies (c).

To see that (c) implies (a), it suffices to show that if $(f+t g) \in E S\left(k[t] / t^{n}\right)$, for all $n$, then $(f+\epsilon g)$ is equisingular via trivial sections. This follows from the usual induction by the next

Lemma 8.3. Suppose $r>1$ and $(f)$ does not define a node; let $g \in k[[X, Y]]$. If $(f+\operatorname{tg}) \in k[[X, Y, t]]$ is equimultiple along the $k[[t]]$-section defined by $I=$ $(X-a(t), Y-b(t))$ (i.e., one gets equimultiple liftings and sections on all truncations $\left.k[[t]] / t^{n}\right)$, then $g \in(X, Y)^{r}$ and either

(i) $a(t)=b(t)=0$ or

(ii) $f$ is a product of two regular parameters with the same tangent direction.

If $(f+t g)$ induces equisingular liftings, then $a(t)=b(t)=0$.

Proof. Proposition 1.6 implies $(f+t g) \in I^{r}$ in $k[[X, Y, t]]$. Differentiation with respect to $t$ yields $g \in I^{r-1}$, whence $f, g \in I^{r-1}$. Let

$$
P=\{b \in k[[X, Y]] \mid b(a(t), b(t)) \equiv 0\} ;
$$

then $P=R \cap I$ is prime. If $P=(X, Y)$, then $a(t)=b(t)=0$, and $g \in(X, Y)^{r}$.

The only other possibility is $P=(b)$, where $b$ is irreducible; write $f=f_{1} b$, $\boldsymbol{g}=g_{1} b$. Since $b$ does not divide $f_{1}$, we have $b \in I^{r-1} ;$ if $r \geq 3$, differentiation 
yields $b_{X}, b_{Y} \in P$ have lower degree. Thus, $r=2$. If $f_{1}$ is a constant, then $(f+t g)=(b)=(f)$, whence $b_{X}, b_{Y} \in I \cap R$, a contradiction. Thus, $f_{1}$ and $b$ are regular parameters with the same tangent direction (since $V(f)$ is not a node). Assuming (after change of coordinates) that $b=Y$, we have that $Y\left(f_{1}+\operatorname{tg} g_{1}\right) \epsilon$ $(X-a(t), Y-b(t))^{2}$, whence $b(t)=0$ and $f_{1}+t g_{1} \in(X-a(t), Y)$. But if $f_{1}(a(t), 0)+\operatorname{tg}_{1}(a(t), 0)=0$, then $g_{1}$ has no constant term, since the linear term of $f_{1}$ is a multiple of $Y$. Consequently, $g=g_{1} b \in(X, Y)^{2}$.

If $(f)$ is as in case (ii), we claim $(f)$ has no nontrivial equisingular liftings; then $(f+t g)$ equisingular would imply, for all $n$, there is an element $\sigma_{n} \epsilon$ $G\left(k[t] / t^{n}\right)$ such that $\left(\sigma_{n}(f)\right)=(f+t g)$ in $R \otimes k[t] / t^{n}$. A small computation would then show $g \in(f)$, whence $(f+t g)=(f)$; but Theorem 3.2 says $(f)$ is equisingular along only the trivial section in $R \otimes k[t] / t^{n}$, whence $a(t)=b(t)=0$.

Now, after a change of coordinates we may suppose $f=Y\left(Y+X^{n}\right)$, some $n \geq 2$. One checks any first-order lifting is equivalent to one of the form $(f+$ $\left.\epsilon \bar{\Sigma}_{i=0}^{2 n-2} a_{i} X^{i}\right)$; if one such is equisingular (with $\left.a_{i} \neq 0\right)$, then so is $\left(f+\epsilon X^{2 n-2}\right)$, since $I$ is an ideal. However, an easy induction on $n$ shows this is false.

Corollary 8.4. Let $g_{1}, \cdots, g_{s} \in I^{\prime}$ be equisingular via trivial sections and induce a basis of $I^{\prime} \bmod \left(f, f_{X}, f_{Y}\right)$. Then $\left(f+\Sigma t_{i} g_{i}\right) \subset k\left[\left[X, Y, t_{1}, \cdots, t_{s}\right]\right]$ defines a versal formal deformation for $\overline{E S}^{\prime}$.

Corollary 8.5. A formal versal equisingular deformation of $Y^{p}+X^{q}(p \leq q)$ is defined by the formal family $\left(Y^{p}+X^{q}+\Sigma t_{i j} X^{i} Y^{j}\right)$ over $k\left[\left[t_{i j}\right]\right]$, where we consider pairs $(i, j)$ with $p i+q j \geq p q, i \leq q-2, j \leq p-2$.

Proof. Follows from Proposition 6.6 and the Theorem.

(8.6) The formal lifting $Y\left(Y-X^{3}+t X^{2}\right)$ is equimultiple along $(X-t, Y)$ as well as along $(X, Y)$; thus, the phenomenon in (ii) of Lemma 8.3 does occur.

(8.7) In the general case, choose $g_{1}, \cdots, g_{s} \in I$ inducing a basis of $\overline{E S}(k[\epsilon])$, and then let $g_{s+1}, \cdots, g_{m} \in k[[X, Y]]$ be such that $g_{1}, \cdots, g_{m}$ induce a basis of $k[[X, Y]] /\left(f, f_{X}, f_{Y}\right)=D(k[\epsilon])$.

Proposition 8.8. Under the above conditions, a formal versal equisingular deformation is given over $k\left[\left[t_{1}, \cdots, t_{s}\right]\right]$ by a lifting of the form $f+\sum_{i=1}^{m} a_{i}(t) g_{i}$, where $\alpha_{i}(t)=\alpha_{i}\left(t_{1}, \cdots, t_{m}\right)$ bas linear term $t_{i}$ if $i \leq s$, and bas no linear term if $i>s$.

Proof. The result follows easily from the following lemma, applied to $\overline{E S} \subset D$.

Lemma 8.9. Let $F_{1} \subset F_{2}$ be an inclusion of versal functors, where $b_{s} \rightarrow F_{1}$ and $b_{T} \rightarrow F_{2}$ are the smootb morphisms yielding versality. Then there exists a (nonunique) surjection $T \rightarrow S$ sucb that the induced inclusion $b_{S} \rightarrow b_{T}$ yields a commutative diagram 


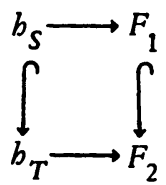

Proof. Let $S_{n}=S / m_{S}^{n}$. The canonical elements of $b_{S}\left(S_{n}\right)$ induce a compatible sequence of elements in $F_{2}\left(S_{n}\right)$. Since $b_{T}\left(S_{n+1}\right) \rightarrow b_{T}\left(S_{n}\right) \times_{F_{2}\left(S_{n}\right)} F_{2}\left(S_{n+1}\right)$ is surjective, we may lift these elements compatibly to elements in $b_{T}\left(S_{n}\right)$; but $T$ is complete, so this process yields a homomorphism $T \rightarrow S$. If now $a \in b_{S}(A)$, some $A \in \underline{C}$, then $a$ is the image of the canonical element in a map $b_{s}\left(S_{n}\right) \rightarrow$ $b_{s}(A)$. Commutativity of the above diagram follows easily. Since $b_{s}(k[\epsilon])=$ $F_{1}(k[\epsilon]) \subset F_{2}(k[\epsilon])=b_{T}(k[\epsilon])$, we have $T \rightarrow S$ is surjective, whence $b_{S} \subset b_{T}$.

Remark 8.10. One cannot in general eliminate terms $g_{i}$ with $i>s$, as seen by the formal equisingular deformation given by

$$
\left[\left(X^{4}-Y^{4}\right)+t\left(X^{4}+X^{3} Y+X^{2} Y^{2}+X Y^{3}\right)\right]^{2}-X^{10} \quad \text { (Example 6.8). }
$$

9. Equisingularity and topological equival ence.

(9.1) In this section we prove that $\pi_{1}$ of the complement of a plane algebroid curve remains constant during formal equisingular lifting. This result is the algebraic analogue of the fact that two equisingular curves over the complex numbers yield knots of the same knot type in $\mathrm{R}^{3}$. Zariski has proved an analogous result for convergent power series rings, relying heavily on topological arguments [12, Theorem 6.1].

We need to use a theorem on deformations of branched covers. Recall that if (d) $\subset R, R$ any domain, a lifting $(\bar{d}) \subset R \otimes A$ is called trivializable if there is a $\sigma \in G(A)$ such that $(\sigma(\bar{d}))=(d)$. If $R \rightarrow S$ is finite and free, then a deformation $R_{A} \rightarrow \bar{S}$ is called trivializable if there is a $\sigma \in G(A)$ such that $\sigma\left[R_{A} \rightarrow \bar{S}\right]$ is the class of the trivial deformation $R_{A} \rightarrow S_{A}$. For details, see [8, $\$ 1.3$ and 1.4$]$.

Theorem A [8, Theorem 2.4.1]. Let $R$ be a regular excellent $k$-domain, $S$ normal, and $R \rightarrow S$ a finite free bomomorphism with discriminant $(d) \subset R$. Then to every trivializable lifting $(\bar{d})$ of $(d)$ to $R_{A}$, there exists a trivializable deformation $R_{A} \rightarrow \bar{S}$ with discriminant $(\bar{d})$, and this deformation is unique up to unique $R_{A}$-isomorphism.

The whole point of Theorem $A$ is the uniqueness of the trivializable deformation class. Recall that if $R$ is a finitely generated $k$-domain, then the trivializable liftings of $(d)$ are precisely those $(\bar{d})$ that do not change the formal nature of the singularities of $R /(d)$.

(9.2) If $f \in k[[X, Y]]=R$ and $F \in k\left[\left[X, Y, t_{1}, \cdots, t_{s}\right]\right]$ are reduced such that $F(X, Y, 0, \cdots, 0)=f(X, Y)$, then we say $V(F)$ defines a formal equisingular lifting of $V(f)$ if the image $F_{n}$ of $F$ in $k\left[\left[X, Y, t_{1}, \cdots, t_{s}\right]\right] /\left(t_{1}, \cdots, t_{s}\right)^{n}$ defines 
an equisingular lifting in the usual sense, for all $n$. Let $S=k\left[\left[t_{1}, \cdots, t_{s}\right]\right]$ and $S_{n}=S / m_{S}^{n}$, and denote by $\sigma_{n}: S_{n}[[X, Y]] \rightarrow S_{n}$ the equisingular section for $V\left(F_{n}\right) \subset$ Spec $S_{n}[[X, Y]]$. Since equisingular sections are unique, the $\sigma_{n}$ are induced by a unique section $\sigma: S[[X, Y]] \rightarrow S$. By Proposition $1.6, F$ is equimultiple along $\sigma$ in the sense that $F \in(\operatorname{Ker} \sigma)^{r}, r=$ multiplicity of $f$. Let $\bar{D}_{1}: \bar{B}_{1} \rightarrow \operatorname{Spec} S[[X, Y]]$ be the blowing-up along $\sigma$. Then, as for artinian $S$, the reduced total transform $R(F)$ of $V(F)$ is obtained by taking $r-1$ copies of the exceptional subscheme of $\bar{B}_{1}$ out of $\bar{D}_{1}^{-1}(V(F))$. Further, $R(F) \subset \bar{B}_{1}$ yields formal equisingular liftings of the singularities of $R(f) \subset B_{1}$. Thus we may repeat the process and obtain a sequence of blowings-up $\bar{D}: \bar{B} \rightarrow$ Spec $S[[X, Y]]$ such that the reduced total transform of $V(F)$ has only ordinary double points as singularities; this will of course induce the same situation on the truncations $p_{n}: B_{n} \rightarrow \operatorname{Spec} S_{n}[[X, Y]]$. Therefore, this definition of formal equisingular lifting agrees with Zariski's via [11, Theorem 7.4].

(9.3) Let $R\left(F_{n}\right) \subset B_{n}$ be the reduced total transform of $F_{n}$ via $p_{n}$. Recall (4.5) that $p_{n}$ is induced via flat base change from a morphism $r_{n}: C_{n} \rightarrow$ Spec $S_{n}[X, Y]$, where $C_{n}$ is an algebraic $k$-scheme, whence $B_{n} \rightarrow \operatorname{Spec} S_{n}$ is a locally trivial deformation of $B \rightarrow$ Spec $k$ in the Zariski topology. In order to apply Theorem $A$, we will have to know $R\left(F_{n}\right) \subset B_{n}$ is locally a trivializable lifting of $R(f)$; since our definition of equisingularity guarantees only formal trivializability, an algebraicity result is needed. But an old result (e.g., [2, Theorem B]) implies that after change of coordinates in $k[[X, Y]]$, the ideal $(f)$ is generated by a polynomial (which we call $f$ ). Further, if $g_{1}, \cdots, g_{m}$ are polynomials inducing a basis of $D(k[\epsilon])$, then $\left(f+\Sigma s_{i} g_{i}\right) \subset k\left[\left[X, Y, s_{1}, \cdots, s_{m}\right]\right]$ defines a formal versal deformation of $k[[X, Y]] /(f)$. Thus, after an $S$-automorphism of $S[[X, Y]]$ inducing the identity $\bmod m_{S},(F)$ is generated by an element in $k[X, Y]\left[\left[T_{1}, \ldots, T_{s}\right]\right]$. Therefore, $R\left(F_{n}\right) \subset B_{n}$ is induced by the reduced total transform in $C_{n}$ of $F_{n}$, considered as an element of $S_{n}[X, Y]$. Since formally trivializable implies locally trivializable for liftings on an algebraic $k$-scheme $[8,2.1 .5]$, it follows that the liftings $R\left(F_{n}\right) \subset B_{n}$ of $R(f)$ are trivializable locally in the Zariski topology.

Theorem 9.4. Let $F \in k\left[\left[X, Y, t_{1}, \cdots, t_{s}\right]\right]$ define a formal equisingular lifting of $f(X, Y)=F(X, Y, 0, \ldots, 0)$. Then the natural map of algebraic fundamental groups

$$
\pi_{1}(\operatorname{Spec} k[[X, Y]]-V(f)) \rightarrow \pi_{1}\left(\operatorname{Spec} k\left[\left[X, Y, t_{1}, \cdots, t_{s}\right]\right]-V(F)\right)
$$

is an isomorphism.

Proof. We must show that every finite étale cover of Spec $k[[X, Y]]-V(f)$ lifts uniquely to an étale cover of Spec $S[[X, Y]]-V(f)$. We shall do this via the natural isomorphisms

$$
\bar{B}-R(F) \simeq \operatorname{Spec} S[[X, Y]]-V(F) \text { and } B-R(f) \simeq \operatorname{Spec} k[[X, Y]]-V(f) \text {. }
$$


Let $X$ be a finite étale cover of $B-R(f)$, and let $Y$ be the normalization of $B$ in the quotient field of $X$. Then $Y \rightarrow B$ is finite and flat (since two-dimensional normal local rings are Cohen-Macaulay), and has its discriminant $D$ supported on a subset of $R(f)$. There is a unique locally trivializable lifting of $D$ to a Cartier divisor $D_{n} \subset B_{n}$ such that $D_{n} \subset R\left(F_{n}\right)$, since $D$ consists of components of $R(f)$ counted with various multiplicities. Since $k[[X, Y]]$ is excellent, so is $B$; the $B_{n}$ 's are locally trivial deformations of $B$, so Theorem $A$ implies there is a (unique) locally trivializable deformation $Y_{n} \rightarrow B_{n}$ with discriminant $D_{n}$, for all $n$.

View $\mathcal{O}_{Y_{n}}$ as a (finite flat) $\mathcal{O}_{B_{n}}$-algebra, and note that $\overline{\bar{p}}: \bar{B} \rightarrow \operatorname{Spec} s[[X, Y]]$ is proper. The Grothendieck existence theorem [EGA, III. 5.1.4] implies there is a unique finite $\theta_{\bar{B}}$-algebra $\theta_{\bar{Y}}$ inducing the $\theta_{Y_{n}}$. For, one first lifts the $\theta_{Y_{n}}$ to $\Theta_{Y}$ as an $\Theta_{\bar{B}}$-module, and then applies the Grothendieck existence theorem again to the algebraic structure maps $\theta_{Y_{n}} \otimes \theta_{Y_{n}} \rightarrow \theta_{Y_{n}}$ to make $\theta_{Y}$ an $\theta_{\bar{B}}$-algebra. An easy local argument shows the corresponding finite map $\bar{Y} \rightarrow \bar{B}$ is flat, whence $\bar{Y} \rightarrow \bar{B}$ is étale off the discriminant divisor $\bar{D}$. But since $\bar{Y} \rightarrow \bar{B}$ induces $Y_{n} \rightarrow$ $B_{n}$, it follows that $\bar{D}$ induces $D_{n}$ for all $n$, so $\bar{D} \subset R(\bar{F})$. Consequently, $\bar{Y}$ induces a finite étale cover of $\bar{B}-R(\bar{F})$ lifting $X \rightarrow B-R(f)$.

For uniqueness, suppose $\bar{X}_{i} \rightarrow \bar{B}-R(\bar{F}), i=1,2$, are finite étale covers lifting $X$. Let $\bar{Y}_{i}$ be the normalization of $\bar{B}$ in the function field of $\bar{X}_{i}$; then $\bar{Y}_{i}$ $\rightarrow \bar{B}$ is finite, and Lemma 9.5 below shows it is flat as well. It suffices to prove $\bar{Y}_{1}$ and $\bar{Y}_{2}$ are isomorphic over $\bar{B}$, or (by the Grothendieck existence theorem again) that the induced $\bar{Y}_{i, n}$ are (compatibly) isomorphic over the $B_{n}$. But $\bar{Y}_{i, n}$ $\rightarrow B_{n}$ is finite, flat, and étale off $R\left(F_{n}\right)$; one also checks that these maps induce $Y \rightarrow B$ over Spec $k$. Since the discriminants involved are liftings of $D_{1}=D \subset R(f)$, they are locally trivializable liftings. Therefore, everything will follow from Theorem $A$ once we show the deformations $\bar{Y}_{i, n} \rightarrow B_{n}$ are locally trivializable, or even formally trivializable $[8,2.1 .6]$; in fact, by $[8,2.4 .5]$, it suffices to check this over nonsingular points $x$ of $D$. However, $\bar{Y}_{i} \rightarrow \bar{B}$ induces a finite free extension of $\hat{\mathcal{O}}_{\bar{B}, x}$ branched along a regular parameter; by Abhyankar's lemma, this extension is obtained by extracting an $m$ th root of a parameter. Therefore, the induced branched covers of $\hat{\mathcal{O}}_{B_{n}, x}$ are trivializable.

Lemma 9.5. Let $k\left[\left[X_{1}, \cdots, X_{n}\right]\right] \rightarrow S(n \geq 2)$ be a finite injective map, with $S$ normal, such that the map on the spectra is étale over Spec $k\left[\left[X_{1}, \cdots, X_{n}\right]\right]-$ $V\left(X_{1} X_{2}\right)$. Then $k\left[\left[X_{1}, \cdots, X_{n}\right]\right] \rightarrow S$ is flat.

Proof. By one form of Abhyankar's lemma (e.g., [8, 2.3.3]),

$$
\pi_{1}\left(\operatorname{Spec} k\left[\left[X_{1}, \ldots, X_{n}\right]\right]-V\left(X_{1} X_{2}\right)\right) \simeq \hat{Z} \oplus \hat{Z} \text {. }
$$


Thus, every such cover is obtained by taking a certain finite free map $k\left[\left[X_{1}, X_{2}\right]\right]$ $\rightarrow S_{1}$, étale off $V\left(X_{1} X_{2}\right)$, and considering the étale cover induced off $V\left(X_{1} X_{2}\right)$ by $k\left[\left[X_{1}, \cdots, x_{n}\right]\right] \rightarrow \bar{S}=S_{1}\left[\left[X_{3}, \cdots, X_{n}\right]\right]$. Since $S_{1}$ is normal, so is $\bar{S}$; since $\bar{S}$ agrees with $S$ off $V\left(X_{1} X_{2}\right)$, then $\bar{S}=S$, whence it is flat over $k\left[\left[X_{1}, \cdots, X_{n}\right]\right]$.

10. A counterexample.

(10.1) We had originally hoped to prove Theorem 9.4 by showing that if $k[[X, Y]] \rightarrow T$ is finite and flat with discriminant $(d)$, and if $(\bar{d}) \subset A[[X, Y]]$ is an equisingular lifting (so $(d)$ should be reduced), then there is a finite flat cover $A[[X, Y]] \rightarrow \bar{T}$ with discriminant $(\bar{d})$ lifting the original "branched cover". The problem is that, in the notation of the proof of Theorem 9.4, $\Gamma(\bar{Y})$ will be a finite normal $\Gamma(\bar{B})=S[[X, Y]]$-algebra, but it will not be flat in general; equivalently, $\Gamma(\bar{Y}) \rightarrow \Gamma(Y)$ need not be surjective (see $[9$, p. 69]). We outline a counterexample suggested by Mumford, in which $(d)$ is an ordinary multiple point; for details, see [9, p. 152].

(10.2) We start with a family of nonsingular space curves $C \subset P^{n} \times X$ over $X$, a nonsingular affine variety, with the following "jump phenomenon" at a closed point $x \in X$; the natural map $\Gamma\left(\mathcal{C}, \mathcal{\Theta}_{\mathcal{C}}(1)\right) \otimes_{\Theta_{X}} k(x) \rightarrow \Gamma\left(C_{x}, \mathcal{O}_{C_{x}}(1)\right)$ is not surjective. To see that such families exist, let $C$ be a nonhyperelliptic curve of genus $g \geq 5$. Then the canonical bundle $K$ is very ample via $C \rightarrow \operatorname{Proj}\left(H^{0}(X, K)^{-}\right)$ $=\mathrm{P}^{8-1}$; further, for generic hyperplane $L \subset H^{0}(X, K)$, any choice of $g-1$ independent sections induces a closed immersion $C \rightarrow \mathrm{P}^{8-2}$. Let $S$ be the $(2 g-2)$ th symmetric power of $C$, the variety parametrizing effective divisors of degree $2 g-$ 2 ; let $J$ be the component of the Picard group of $C$ parametrizing invertible sheaves of degree $2 g-2, \Phi: S \rightarrow J$ the natural map, and $x \in J$ the point corresponding to $K$. A divisor $D \in|K|$ corresponds to a point $s \in \Phi^{-1}(x)$. One can identify the tangent space map $\Phi_{*}: T_{S, s} \rightarrow T_{J, x}$ with the map on cohomology $H^{0}\left(N_{D}\right) \rightarrow$ $H^{1}\left(\Theta_{X}\right)[5$, p. 165], whence it has rank $g-1$. Therefore, one can find near $s$ a nonsingular $(g-1)$-dimensional variety $V_{s}$ such that $\Phi \mid V_{s}$ is a closed immersion; let $W_{s}$ be the image. We may view $V_{s}$ as a global section of the invertible sheaf on $C \times W_{s}$ induced from the universal invertible sheaf $M$ of degree $2 g-2$ on $C \times J$; this section extends $s$. Thus, if $s_{1}, \cdots, s_{g-1} \in H^{0}(X, K)$ induce a projective embedding $C \rightarrow \mathrm{P}^{g-2}$, we may intersect the $W_{s_{i}}$ near $x$, and find a nonsingular curve $X$ through $x$ on $J$ over which the sections $s_{i}$ extend. Shrinking $X$ perhaps, we get a family of closed immersions $r: C \times X \rightarrow \mathbf{P}^{8-2} \times X$ such that $r^{*}(\Theta(1))$ is the invertible sheaf on $C \times X$ induced from $M$ on $C \times J$. Using Riemann-Roch, one checks that this family of embeddings has the desired jump phenomenon at $x \in X$.

(10.3) Denote by $C$ the embedding of $C_{x}$ in $\mathbf{P}^{n}$, and let $f_{0}: C \rightarrow \mathrm{P}^{1}$ be a "generic projection" onto a line; thus, if $C$ has degree $d$ in $\mathrm{P}^{n}$ and genus $g$, there are $b=2(g+d-1)$ distinct branch points on $\mathbf{P}^{1}$. Shrinking $X$ perhaps, we 
may extend to a family of generic projections $f: C \rightarrow \mathrm{P}^{1} \times X$, where the discriminant of $f$ is a finite étale cover of $X$ of degree $b$. Writing $X=\operatorname{Spec} S$, and supposing $m \subset S$ corresponds to $C$, consider the induced map of affine cones (see [EGA, II.8.8]):

$$
\begin{gathered}
\bigoplus_{i=0}^{\infty} \Gamma\left(\mathbf{P}^{1} \times X, \mathcal{O}_{\mathbf{P}^{1} \times X}(i)\right) \rightarrow \bigoplus_{i=0}^{\infty} \Gamma\left(\mathcal{C}, \mathcal{O}_{\mathcal{e}}(i)\right) . \\
\|[X, Y]
\end{gathered}
$$

(10.4) One checks that $S[X, Y] \rightarrow \bar{T}$ is a finite map of normal domains, and it is étale off a subscheme $Z \subset \operatorname{Spec} S[X, Y]$ which, over Spec $S$, yields a family of ordinary $b$-tuple points. But $\bar{T} / m \bar{T} \rightarrow \bigoplus_{i=0}^{\infty} \Gamma\left(C, \mathcal{O}_{C}(i)\right)=T$ is a strict inclusion (by the jump phenomenon) and a finite birational map (since $\Gamma\left(\mathcal{C}, \mathcal{\Theta}_{\mathcal{C}}(i)\right) \rightarrow$ $\Gamma\left(C, \mathcal{O}_{C}(i)\right)$ is surjective for all large $\left.i\right)$; since $T$ is normal, $\bar{T} / m \bar{T}$ is not normal. Then $g:$ Spec $\hat{T} \rightarrow$ Spec $k[[X, Y]]$ (taking completions over the vertex of the cone) is a finite flat morphism whose discriminant $(d)$ is an ordinary multiple point of $\leq b$ branches; let $\left(d_{n}\right) \subset \operatorname{Spec} S / m^{n}[[X, Y]]$ be the equisingular lifting induced by $Z$. Then $g$ cannot lift for all $n$ to a cover with discriminant $\left(d_{n}\right)$. If it did, there would be a finite flat map Spec $T_{1} \rightarrow \operatorname{Spec} \hat{S}[[X, Y]]$, isomorphic off the pull-back of $Z$ to Spec $\hat{\bar{T}} \rightarrow \operatorname{Spec} \hat{S}[[X, Y]]$, and inducing $g$. But since $\hat{\bar{T}}$ is normal, there would have to be a factorization $\hat{S}[[X, Y]] \rightarrow T_{1} \rightarrow \hat{\bar{T}}$; but reduction mod the maximal ideal of $\hat{S}$ yields a contradiction.

Added in proof. The first part of the proof of Proposition 6.6 is incomplete as it stands. One must check that if $i+j-p \geq q-p-1$, but $p i+q j<p q$, then the reduced total transform is not equisingular (if $a_{i j} \neq 0$ ), even though one has a trivial deformation of the proper transform. These inequalities occur only if $i+j=$ $q-1$ and $j(q-p)<p$; of course $j \geq 1$, so $p>q-p$. Letting $r=q-p$, we claim $X\left(X^{r}+T^{p}+\epsilon \Sigma a_{j} X^{r-1} T^{j}\right)$ is equisingular only if $a_{j}=0$ for $j<p / r$. Since $E S(k[\epsilon])$ is an ideal, it suffices to show $X\left(X^{r}+T^{p}+\epsilon X^{r-1} T^{s}\right)$ is not equisingular, where $s$ is the largest integer $<p / r$. This is proved by induction on $p r$, by blowing up and treating separately the cases $r\langle p-r, r=p-r$, and $r\rangle p-r$. One uses that $g$ singular, $(f+\epsilon h) \cdot g$ equi singular imply (by 3.2 and 3.4 ) that $f+\epsilon h$ is equisingular along the trivial section.

Conceming $\$ 10$, we have proved that the cover $k[[X, Y]] \rightarrow T$ does lift (with prescribed equisingular deformation of the discriminant), provided $T$ has a singularity for which the "geometric genus" equals the "arithmetic genus of the fundamental cycle" (e.g., if $T$ has a rational singularity). For, it will follow automatically in this case that $\Gamma(\bar{Y}) \rightarrow \Gamma(Y)$ is surjective. 


\section{REFERENCES}

EGA A. Grothendieck, Eléments de géométrie algébrique, Inst. Hautes Études Sci. Publ. Math. Paris 4, 8, 11, 17, 20, 24, 28, 32 (1960-67). MR 29 \#1210; 30 \#3885; 33 \#7330; 36 \#177a, b, c; $36 \# 178 ; 39 \# 220$.

1. - Séminaire de géométrie algébrique, mimeographed notes, Inst. Hautes Études Sci. Paris, 1960-61.

2. H. Hironaka, On the equivalence of singularities. I, Arithmetic Algebraic Geometry (Proc. Conf., Purdue Univ., 1963), Harper \& Row, New York, 1965, pp. 153-200. MR 34 $\# 1317$.

3. S. Lichtenbaum and M. Schlessinger, The cotangent complex of a morphism, Trans. Amer. Math. Soc. 128 (1967), 41-70. MR 35 \#237.

4. J. Milnor, Singular points of complex hypersurfaces, Ann. of Math. Studies, no. 61, Princeton Univ. Press, Princeton, N. J.; Univ, of Tokyo Press, Tokyo, 1968. MR 39 $\# 969$.

5. D. Mumford, Lectures on curves in an algebraic surface, Ann. of Math. Studies, no. 59, Princeton Univ. Press, Princeton, N. J., 1966. MR 35 \#187.

6. M. Schlessinger, Functors of Artin rings, Trans. Amer. Math. Soc. 130 (1968), 208222. MR 36 \# 184 .

7. M. Schlessinger, Infinitesimal deformations of singularities, Thesis, Harvard, 1964.

8. J. Wahl, Deformation of plane curves with nodes and cusps, Amer. J. Math. (to appear).

9. - Deformations of branched covers and equisingularity, Thesis, Harvard, 1971.

10. O. Zariski, Studies in equisingularity. I. Equivalent singularities of plane algebroid curves, Amer. J. Math. 87 (1965), 507-536. MR 31 \#2243.

11. - Studies in equisingularity. II. Equisingularity in codimension one (and characteristic zero), Amer. J. Math. 87 (1965), 972-1006. MR 33 \#125.

12. - Studies in equisingularity. III. Saturation of local rings and equisingularity, Amer. J. Math. 90 (1968), 961-1023. MR 38 \#5775.

DEPARTMENT OF MATHEMATICS, UNIVERSITY OF CALIFORNIA, BERKELEY, CALIFORNIA 94720

INSTITUTE FOR ADVANCED STUDY, PRINCETON, NEW JERSEY 08540

Current address: Department of Mathematics, University of North Carolina, Chapel Hill, North Carolina 27514 